\title{
Optimal Call Admission Control on a Single Link With a GPS Scheduler
}

\author{
Antonis Panagakis, Nandita Dukkipati, Student Member, IEEE, Ioannis Stavrakakis, Senior Member, IEEE, and
} Joy Kuri

\begin{abstract}
The problem of call admission control (CAC) is considered for leaky bucket constrained sessions with deterministic service guarantees (zero loss and finite delay bound) served by a generalized processor sharing scheduler at a single node in the presence of best effort traffic. Based on an optimization process, a CAC algorithm capable of determining the (unique) optimal solution is derived. The derived algorithm is also applicable, under a slight modification, in a system where the best effort traffic is absent and is capable of guaranteeing that if it does not find a solution to the CAC problem, then a solution does not exist. The numerical results indicate that the $\mathrm{CAC}$ algorithm can achieve a significant improvement on bandwidth utilization as compared to a (deterministic) effective bandwidth-based CAC scheme.
\end{abstract}

Index Terms-Call admission control, generalized processor sharing, optimal bandwidth allocation.

\section{INTRODUCTION}

$\mathbf{T}$ HE GENERALIZED processor sharing (GPS) scheduling discipline has been widely considered to allocate bandwidth resources to multiplexed traffic streams. Its effectiveness and capabilities in guaranteeing a certain level of quality of service $(\mathrm{QoS})$ to the supported streams in both a stochastic ([5], [6], [4]) and deterministic ([1]-[3], [7]) sense have been investigated. Traffic management based on deterministic guarantees is expected to lead to lower network resource utilization compared to that under stochastic guarantees. Nevertheless, such considerations are necessary when deterministic guarantees are required by the applications. In addition, they can provide valuable insight and methodology for the consideration of stochastic guarantees.

Under the GPS scheduling discipline traffic is treated as an infinitely divisible fluid. A GPS server that serves $N$ sessions is characterized by $N$ positive real numbers $\phi_{1}, \ldots, \phi_{N}$, referred to as weights. These weights affect the amount of service provided to the sessions (or their bandwidth shares). More specifically, if $W_{i}(\tau, t)$ denotes the amount of session $i$ traffic served in a time interval $(\tau, t]$, then the following relation will hold for any session $i$ that is continuously backlogged in the interval $(\tau, t]$;

Manuscript received November 21, 2001; revised January 8, 2003, and June 17, 2003; approved by IEEE/ACM TRANSACTIONS ON NETWORKING Editor J.-Y. LeBoudec. This work was supported in part by the Special Account for Research Projects of the National and Kapodistrian University of Athens and by the IST Programme under Contract IST-1999-10160 (VideoGateway).

A. Panagakis and I. Stavrakakis are with the Department of Informatics, University of Athens, Athens 157-84, Greece (e-mail: grad0260@di.uoa.gr; istavrak@di.uoa.gr).

N. Dukkipati and J. Kuri are with the Centre for Electronics Design and Technology, Indian Institute of Science, Bangalore 560012, India (e-mail: dnandita@stanford.edu; kuri@cedt.iisc.ernet.in).

Digital Object Identifier 10.1109/TNET.2004.836133 session $i$ is considered to be backlogged at time $t$ if a positive amount of session $i$ traffic is queued at time $t$

$$
\frac{W_{i}(\tau, t)}{W_{j}(\tau, t)} \geq \frac{\phi_{i}}{\phi_{j}}, \quad j=1,2, \ldots N .
$$

In their seminal papers [1], [2] on GPS, Parekh and Gallager have analyzed the GPS scheduling discipline in a deterministic setting where the traffic of each session is regulated by a leaky bucket regulator. In the single-node and the multiple-node cases they obtained closed form expressions for bounds on the delay and backlog for a certain class of GPS schedulers called rate proportional processor sharing (RPPS) schedulers. In [7], Zhang et $a l$. obtained closed-form expressions for the end-to-end performance bounds for a broader class of GPS networks known as consistent relative session treatment (CRST) GPS networks. In [11], Yaron and Sidi studied GPS networks with exponentially bounded burstiness arrivals. In [5], Zhang et al. investigated the behavior of GPS in a stochastic setting. The above-mentioned papers derive delay and backlog bounds for a session in GPS schedulers, given a particular weight allocation for the session.

The inverse problem of mapping the QoS requirements of the sessions to weight allocations in GPS schedulers is of practical importance. Kesidis et al. in [12] and Zhang et al. in [6] address this problem in a stochastic setting (stochastic arrival processes and statistical guarantees) employing packet loss probability as the QoS metric. In [13], the above problem is addressed for leaky bucket regulated connections and statistical delay guarantees. The work most relevant to ours is that of [3], where tight delay bounds (also reported in [9]) have been presented in conjunction with a CAC algorithm for the single-node case, which aims to calculate a weight assignment for the sessions that would not result in an overachievement of session delays. In order to do so, the dependencies among the sessions must be considered. The CAC procedure in [3] does not address this problem directly since it employs an exhaustive search, having performance bound calculations as an intermediate step. More specifically, the maximum delay experienced by the sessions is determined for a weight assignment and the assignment is modified trying to maximize an objective function. While the search in [3] terminates after a finite number of steps, it does not guarantee that an acceptable assignment does not exist if it is not found. This could result in an overallocation of bandwidth and sometimes a call block, even if the bandwidth necessary to guarantee the call's QoS requirements is available.

A major contribution of this paper is a CAC algorithm for the single-node case which fully exploits the bandwidth sharing mechanism of GPS and determines the optimal weights $\phi$ directly from the QoS requirements of the sessions rather than through a recursive computation of the induced delay bounds 
and weight reassignment. In addition, it turns out that the optimal scheme is less complex than that of [3].

The investigation of the CAC problem and the derivation of the optimal solution, although limited to the single-node case, are relevant not only on a theoretical but also on a practical basis. If the bottleneck links in the network are at the edges of the network, it suffices to have an admission control and bandwidth reservation at the access link. For example, in [14] a realistic scenario is described where a high-speed LAN is connected to the high-speed Internet through a $2-\mathrm{Mb} / \mathrm{s}$ access link, which is the bottleneck. As another example, where the single-node case analysis suffices to guarantee the end-to-end delay, consider the input of a (semi-)permanent VP in an ATM network. Assuming that the same amount of bandwidth is reserved for it at all nodes, the end-to-end delay of the sessions is equal to the delay experienced at the first node (traffic is considered as fluid). In addition, the derivation of the optimal scheme for the single-node case, apart from its own theoretical value as a bound on the performance of GPS, provides insight into the bandwidth-sharing mechanism of GPS and sheds light into the difficulties occurring in the multiple-node case.

The major results are derived by considering a mixed traffic environment in which the bandwidth resource controlled by the GPS server is shared by a number of QoS sensitive streams and best effort traffic. This system will be referred to as a best effort traffic aware generalized processor sharing (BETA-GPS) system. The developed algorithm determines the minimum $\phi$ assignments for the QoS sensitive streams which are just sufficient to meet their QoS and, consequently, maximizes the (remaining) $\phi$ assignment to the best effort traffic. Based on the main results, an optimal CAC scheme is proposed in this paper for a decoupled system of GPS-controlled QoS sensitive traffic and best effort traffic (referred to as pure QoS system, see Section IV). The formulation of the pure QoS system facilitates the derivation of the minimum required GPS scheduler capacity to support N QoS sensitive streams, which is, in itself, an interesting problem.

In Section II, some basic definitions are presented and the BETA-GPS environment is described. In Section III, an optimization process for the weight assignment is developed, the properties of the optimal assignment are studied, and the proposed optimal CAC algorithm is derived. In Section IV, we describe how the results of Section III can be utilized in the pure QoS GPS system. In Section V, some numerical results are presented. Section VI concludes the paper.

\section{DEFINITIONS AND DESCRIPTION OF THE BETA-GPS SYSTEM}

\section{A. GPS-Related Definitions}

QoS sensitive sessions will be assumed to be leaky bucket constrained. That is, the amount of session $i$ traffic arriving at the GPS server over any interval $(\tau, t]$, referred to as the session $i$ arrival function $A_{i}(\tau, t)$, will be bounded as follows: $A_{i}(\tau, t) \leq$ $\sigma_{i}+\rho_{i}(t-\tau), \forall t \geq \tau \geq 0 ; \sigma_{i}$ and $\rho_{i}$ represent the burstiness and long-term maximum mean arrival rate of session $i$. A session $i$ is characterized as a greedy starting at time $\tau$ if the aforementioned bound is achieved, that is if $A_{i}(\tau, t)=\sigma_{i}+\rho_{i}(t-\tau), \forall t \geq$ $\tau$. A GPS system busy period is defined to be a maximal time interval during which at least one session is backlogged at any time instant in the interval.

An all-greedy GPS system is defined as a system in which all the sessions are greedy starting at time zero, the beginning of a system busy period. The significance of the all-greedy system follows from [11, Th. 3]: If the input link speed of any session $i$ exceeds the GPS service rate, then for every session $i$, the maximum delay $D_{i}^{*}$ and the maximum backlog $Q_{i}^{*}$ are achieved (not necessarily at the same time) when every session is greedy starting at time zero, the beginning of a system busy period. This implies that if the server can guarantee an upper bound on a session's delay, under the all-greedy system assumption this bound would be valid under any arrival pattern. In view of the previous observation and by examining only all-greedy systems, the CAC problem for a GPS system is simplified.

Let $t=0$ denote the beginning of a system busy period in the all-greedy system. For each session $i$ the arrival function takes the form $A_{i}(0, t)=\sigma_{i}+\rho_{i} \cdot t, \forall t \geq 0$. If $Q_{i}(t)$ denotes the amount of session $i$ traffic queued at time $t$, then $Q_{i}(t)=$ $A_{i}(0, t)-W_{i}(0, t)$ and $Q_{i}(t)=0$ for all $t \leq 0$ by assumption. Let $e_{i}$ denote the backlog clearing time of session $i$, then

$$
e_{i}=\sup \left\{t>0: Q_{i}(t)>0\right\}
$$

and $B_{i}=\left(0, e_{i}\right)$ corresponds to the session $i$ busy period.

The QoS sensitive sessions will be assumed to have a stringent delay requirement, denoted by $D_{i}$ for session $i$. Thus, a QoS sensitive session $i$ will be characterized by the triplet $\left(\sigma_{i}, \rho_{i}, D_{i}\right)$. To ensure that the delay constraint for the QoS sensitive session $i$ is met, a minimum amount of service $N_{i}(0, t)$ must be provided by the GPS server to session $i$ over the interval $(0, t]$, where

$$
N_{i}(0, t)= \begin{cases}\sigma_{i}+\rho_{i}\left(t-D_{i}\right), & t \geq D_{i} \\ 0, & t<D_{i} .\end{cases}
$$

That is, the actual amount of service (work) $W_{i}(0, t)$ provided to session $i$ over the interval $(0, t]$ must satisfy

$$
W_{i}(0, t) \geq N_{i}(0, t), \quad \forall t \geq 0 .
$$

The function $N_{i}(0, t)$ is referred to as session $i$ requirements.

\section{B. Description of the System and Problem Formulation}

The BETA-GPS system is depicted in Fig. 1. The BETA-GPS server capacity $C_{G}$ is assumed to be shared by $N$ QoS sensitive sessions with descriptors $\left(\sigma_{i}, \rho_{i}, D_{i}\right), i=1, \ldots, N$ and best effort traffic represented by an additional session. Each session is provided a buffer and the input links are considered to have infinite capacity. Quantities associated with a QoS sensitive session (best effort session) will be identified by a subscript $i(b e)$, $i=1, \ldots, N$. To avoid degenerate cases and be consistent with the GPS definitions it is assumed that $\sigma_{i} \rho_{i} D_{i} \neq 0, i=1, \ldots, N$ and that the $\phi$ assignment of the BETA-GPS scheduler to a session cannot be zero $\left(\phi_{i}>0, i=1, \ldots, N, b e\right)$.

Generally, the task of CAC is to determine whether the network can accept a new session without causing QoS requirement violations. In the case of a GPS scheduler, it should also provide the server with the weight assignment which will be used in the actual service of the admitted calls. A CAC scheme for a GPS server is considered to be optimal if its incapability to admit a specific set of sessions implies that no $\phi$ assignment 


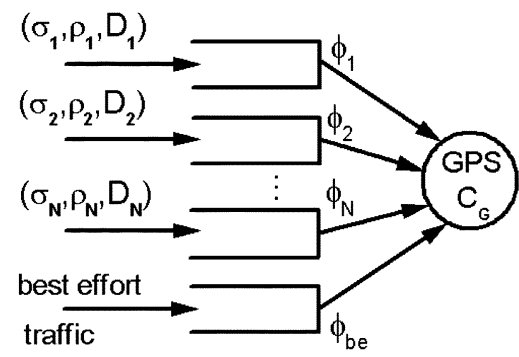

Fig. 1. Functional diagram of the BETA-GPS system.

exists under which the server could serve this set of sessions (respecting all QoS requirements even under the worst case arrival scenario all-greedy system). In addition, an optimal CAC scheme for the BETA-GPS system should seek to maximize the amount of service provided to the (traffic unlimited) best effort session under any arrival scenario and over any time horizon, while satisfying the QoS requirement of the (traffic limited) QoS sensitive sessions. That is, it should seek to maximize the normalized $^{1}$ weight assigned to the best effort traffic $\left(\phi_{b e}\right)$ while satisfying the QoS requirement of QoS sensitive sessions.

Obviously, maximizing the weight assigned to the best effort traffic is equivalent to minimizing the sum of weights assigned to the QoS sensitive sessions. In view of this discussion, the following definition may be provided.

Definition 1: (a) The optimal CAC scheme for the BETA-GPS system is the one that is based on the optimal $\phi$ assignment for the BETA-GPS system.

(b) The optimal $\phi$ assignment for the BETA-GPS system is the one that allows the QoS sensitive sessions to meet their QoS requirements - provided that it is possible - and achieves $\max \left\{\phi_{\mathrm{be}}\right\}=\max \left\{1-\sum_{i=1}^{N} \phi_{i}\right\}$ or, equivalently, $\min \left\{\sum_{i=1}^{N} \phi_{i}\right\}$, where $\phi_{i} \in \mathbb{R}_{+}^{*}, i=1, \ldots, N$, be according to the definition of GPS.

In an all-greedy system, all QoS sensitive sessions are backlogged at time $t=0^{+}$. Let $\mathcal{B}(t)$ denote the set of sessions that are backlogged in the interval $(0, t]$, and let $\mathcal{E}(t)$ denote the set of sessions which have emptied their backlog before time $t$, that is, $\mathcal{B}(t)=\left\{i: e_{i} \geq t, i=1, \ldots, N\right\}, \mathcal{E}(t)=\{i:$ $\left.e_{i}<t, i=1, \ldots, N\right\}$, where $e_{i}$ is defined in (2). Each session $k \in \mathcal{E}(t)$ requires a rate equal to $\rho_{k}$. Consequently, the bandwidth that can be considered to be available for allocation to the sessions $i, i \in \mathcal{B}(t)$ is equal to $\left(C_{G}-\sum_{k \in \mathcal{E}(t)} \rho_{k}\right)$. Session $i \in \mathcal{B}(t)$ will be allocated a share of that bandwidth equal to $\phi_{i}\left(1-\sum_{k \in \mathcal{E}(t)} \phi_{k}\right)^{-1}$ and will be served with a rate $\phi_{i}\left(C_{G}-\sum_{k \in \mathcal{E}(t)} \rho_{k}\right)\left(1-\sum_{k \in \mathcal{E}(t)} \phi_{k}\right)^{-1}$. Let

$$
\hat{C}(t) \triangleq \frac{C_{G}-\sum_{j \in \mathcal{E}(t)} \rho_{j}}{1-\sum_{j \in \mathcal{E}(t)} \phi_{j}}
$$

be referred to as the normalized backlogged sessions allocated (NBSA) bandwidth (this quantity is called "universal slope" in [1]). Clearly, $\hat{C}(t)$ changes value each time a session empties its backlog and remains constant between two consecutive backlog clearing times. Thus, $\hat{C}(t)$ is a piecewise constant function with the discontinuity points coinciding with the backlog clearing times of the sessions.
Let $\left\{\mathrm{b}_{i}\right\}_{i=1}^{L}, L \leq N$ denote the ordered set of distinct backlog clearing times and let $b_{0}=0$ be the beginning of the system busy period. For two consecutive backlog clearing times, $\mathrm{b}_{j-1}$ and $\mathrm{b}_{j}, \hat{C}\left(\mathrm{~b}_{j-1}^{+}\right)=\hat{C}\left(\mathrm{~b}_{j}^{-}\right)$. Treating the NBSA bandwidth as a left continuous function implies that $\hat{C}\left(\mathrm{~b}_{j}\right)=\hat{C}\left(\mathrm{~b}_{j}^{-}\right)$and

$$
\hat{C}(t)=\frac{C_{G}-\sum_{k \in \mathcal{E}\left(\mathrm{b}_{j}\right)} \rho_{k}}{1-\sum_{k \in \mathcal{E}\left(\mathrm{b}_{j}\right)} \phi_{k}} \quad \forall t \in\left(\mathrm{b}_{j-1}, \mathrm{~b}_{j}\right] .
$$

$\hat{C}(t)$ is an increasing function of time since it preserves a constant value between two consecutive backlog clearing times and $\hat{C}\left(\mathrm{~b}_{j}\right)<\hat{C}\left(\mathrm{~b}_{j}^{+}\right)$for a backlog clearing time $\mathrm{b}_{j} .^{2}$

The amount of scheduler's work that is shared among the backlogged sessions $i \in \mathcal{B}\left(\mathrm{b}_{j}\right)$ over the time interval $\left(\mathrm{b}_{j-1}, \mathrm{~b}_{j}\right]$ is equal to $\left(C_{G}-\sum_{i \in \mathcal{E}\left(\mathrm{b}_{j}\right)} \rho_{i}\right)\left(\mathrm{b}_{j}-\mathrm{b}_{j-1}\right)$.

Let

$$
\hat{W}\left(\mathrm{~b}_{j-1}, \mathrm{~b}_{j}\right) \triangleq \hat{C}\left(\mathrm{~b}_{j-1}^{+}\right)\left(\mathrm{b}_{j}-\mathrm{b}_{j-1}\right)
$$

be referred to as the NBSA work. Then, in view of (6) and (7), session $i \in \mathcal{B}\left(\mathrm{b}_{j}\right)$ is allocated an amount of work equal to $\phi_{i} \hat{W}\left(\mathrm{~b}_{j-1}, \mathrm{~b}_{j}\right)$ over $\left(\mathrm{b}_{j-1}, \mathrm{~b}_{j}\right]$.

\section{Optimal CAll Admission CONTROL fOR THE BETA-GPS SYSTEM}

\section{A. Optimizing an Acceptable $\phi$ Assignment}

In this section, a process that converts an acceptable $\phi$ assignment into a more efficient acceptable one is developed. An acceptable $\phi$ assignment is one which is feasible (that is, $\left.\sum_{i=1}^{N} \phi_{i}<1\right)$ and delivers the required QoS to each of the supported QoS sensitive sessions. A $\phi$ assignment is more efficient than another if the sum of $\phi \mathrm{s} \sum_{i=1}^{N} \phi_{i}$ under the former assignment is smaller than that under the latter.

The aforementioned process will be referred to as the eXpand Minimum busy period First (XMF) process. According to the XMF process, each QoS sensitive session's busy period is expanded as much as its QoS would permit, starting from the set of QoS sensitive sessions that empty their backlog first in order. A very important property of the XMF process is that it converts any acceptable $\phi$ assignment into the optimal one.

Let $\Pi$ denote the set of acceptable policies (or equivalently, $\phi$ assignments) and let $\pi_{a} \in \Pi$. The application of the XMF process to $\pi_{a}$ results in an acceptable policy $\pi_{o}=\operatorname{XMF}\left(\pi_{a}\right)$,

$$
\begin{aligned}
& { }^{2} \text { Proof: Assume, without loss of generality, that only one session, session } \\
& k \text {, empties its backlog at } \mathrm{b}_{j} \text {. The fact that session } k \text { empties its backlog at } \mathrm{b}_{j} \\
& \text { implies that it was served with a rate greater than } \rho_{k} \text { at } t=\mathrm{b}_{j}^{-}, \text {i.e., } \\
& \qquad \begin{aligned}
\rho_{k} & <\phi_{k} \hat{C}\left(\mathrm{~b}_{j}^{-}\right) \Leftrightarrow \rho_{k} \overline{\mathcal{P}}_{j}<\phi_{k} \overline{\mathcal{R}_{j}} \Leftrightarrow \overline{\mathcal{P}_{j}} \overline{\mathcal{R}_{j}}-\rho_{k} \overline{\mathcal{P}}_{j} \\
& >\overline{\mathcal{P}}_{j} \overline{\mathcal{R}_{j}}-\phi_{k} \overline{\mathcal{R}_{j}} \Leftrightarrow \overline{\mathcal{P}}_{j}\left(\overline{\mathcal{R}}_{j}-\rho_{k}\right) \\
& >\left(\overline{\mathcal{P}}{ }_{j}-\phi_{k}\right) \overline{\mathcal{R}}_{j} \Leftrightarrow \overline{\mathcal{P}}_{j} \overline{\mathcal{R}}_{j}^{+}>\overline{\mathcal{P}}_{j}^{+} \overline{\mathcal{R}}_{j} \Leftrightarrow \hat{C}\left(\mathrm{~b}_{j}\right) \\
& =\hat{C}\left(\mathrm{~b}_{j}^{-}\right)<\hat{C}\left(\mathrm{~b}_{j}^{+}\right)
\end{aligned}
\end{aligned}
$$

where

$$
\begin{aligned}
& \overline{\mathcal{R}_{j}}=C_{G}-\sum_{i \in \mathcal{E}\left(\mathrm{b}_{j}\right)} \rho_{i}, \quad \overline{\mathcal{P}}_{j}=1-\sum_{i \in \mathcal{E}\left(\mathrm{b}_{j}\right)} \phi_{i} \\
& \overline{\mathcal{R}}_{j}^{+}=C_{G}-\sum_{i \in \mathcal{E}\left(\mathrm{b}_{j}^{+}\right)} \rho_{i}, \quad \overline{\mathcal{P}}_{j}^{+}=1-\sum_{i \in \mathcal{E}\left(\mathrm{b}_{j}^{+}\right)} \phi_{i} .
\end{aligned}
$$

${ }^{1}$ Without loss of generality, it is assumed that $\sum_{i=1}^{N} \phi_{i}+\phi_{\mathrm{be}}=1$. 
which is not less efficient than $\pi_{a} ; \mathrm{XMF}(\pi)$ denotes a policy that is generated by applying the XMF process to $\pi$. In particular, it will be shown that $\pi_{o}$ is unique and more efficient than $\pi_{a}$, except for the case in which $\pi_{a}=\pi_{o}$.

Let ${ }^{a} \mathrm{I}_{k}$ denote the set of QoS sensitive sessions that empty their backlog $k$ th in order under $\pi_{a}$, and let ${ }^{a} \mathrm{~b}_{k}$ be the time instant when this happens. Let ${ }^{a} \mathrm{I}_{k}^{P}=\bigcup_{s=1}^{k-1}{ }^{a} \mathrm{I}_{s}$ and ${ }^{a} \mathrm{I}_{k}^{F}=$ $\bigcup_{s>k+1}{ }^{a} \mathrm{I}_{s}$ denote the sets of sessions that empty their backlog before (past) and after (future) ${ }^{a} \mathrm{~b}_{k}$, respectively. The following definitions will be needed.

\section{Definition 2:}

a) A session $i$ is compressed (decompressed) in $\phi$ space if its weight is decreased (increased).

b) A session $i$ is decompressed in $t$ space, or its busy period is expanded, if its backlog clearing time is increased.

c) Sessions in ${ }^{a} \mathrm{I}_{k}$ are uniformly decompressed in $t$ space, or their busy periods are uniformly expanded, if their backlog clearing times are equally increased.

d) A session $i$ preserves its position in $\phi(t)$ space if its weight (backlog clearing time) remains unchanged.

e) A set $A \subseteq{ }^{a} \mathrm{I}_{k}$ is compressible in $\phi$ space if $\forall i \in A d \phi_{i}>$ 0 exists such that sessions $i \in A$ do not violate their delay bounds when they are assigned a weight $\phi_{i}-d \phi_{i}$, under the conditions: a) sessions in ${ }^{a} \mathrm{I}_{k}^{P} \cup\left\{{ }^{a} \mathrm{I}_{k} \backslash A\right\}$ preserve their position in $\phi$ space and $\mathrm{b}$ ) sessions which emptied their backlog after sessions in $A$ still empty their backlog after sessions in $A$.

1) Description of the XMF Process: The XMF process applied to an acceptable policy $\pi_{a} \in \Pi$ is described in Fig. 2. Throughout the description only the treatment of the QoS sensitive sessions is considered, and this is sufficient since the weight assigned to best effort traffic is given by $1-\sum_{i=1}^{N} \phi_{i}^{\pi}$ under a policy $\pi$.

At this point, the following should be noted. XMF is a conceptual process which is not directly applicable at a computational level. Sessions' weights and busy periods change in a continuous way under this process. In addition, and in order to keep the presentation clear and simple, each time that the process modifies the policy it is applied to, the process is presented to be reapplied in its entirety to the modified policy, although not necessary. The rationale for this approach is that since XMF is a conceptual process, it is not a concern how many times it will be applied as long as it terminates (generates results) after a finite number of steps.

2) Properties of the XMF Process: The XMF process forces all sessions to empty their backlog as late as possible. The only parameters that impose an upper limit on the expansion of the busy periods are the sessions' delay bounds, which do not depend on $\pi_{a}$. Thus, one could expect $\pi_{o}$ not to depend on $\pi_{a}$. The following propositions hold. Their proofs may be found in the Appendix.

Proposition 1: The (intermediate) policy $\pi_{b}$ that is defined at the end of Steps II-1a), II-1b), or II-2a) is acceptable and more efficient than $\pi_{a}$.

Proposition 2: The final policy that results when the application of the XMF process to an arbitrary original acceptable policy is terminated is acceptable and does not depend on the original policy; that is, $\forall \pi_{a 1}, \pi_{a 2} \in \Pi, \operatorname{XMF}\left(\pi_{a 1}\right)=$ $\operatorname{XMF}\left(\pi_{a 2}\right)=\pi_{o}, \pi_{o} \in \Pi$.

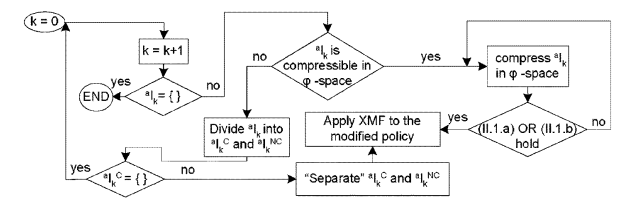

(0) Initially, $k=0$.

(I) $\mathrm{k}=\mathrm{k}+1$. If ${ }^{a} \mathrm{I}_{k}=\emptyset$ goto (III)

(II) Sessions in ${ }^{a} \mathrm{I}_{k}$ are considered. Sessions in ${ }^{a} \mathrm{I}_{k}^{P}$ preserve their position in $\phi$ - space. This implies that the sessions in ${ }^{a} \mathbf{I}_{k}^{P}$ preserve their position in $t$ - space as well ${ }^{\dagger}$.

(II.1) If ${ }^{a} I_{k}$ is compressible the sessions in ${ }^{a} I_{k}$ are compressed in $\phi$ - space in such a way that their busy periods are uniformly expanded. At the same time the sessions in ${ }^{a} \mathrm{I}_{k}^{F}$ are decompressed in $\phi$ - space in such a way (under the condition) that they receive the same amount of work up to the end of the (modified) busy periods of sessions in ${ }^{a} \mathbf{I}_{k}$ as they did under $\pi_{a}$. This "conditional exchange of weights" is possible ${ }^{\dagger}$ and does not alter the backlog clearing times of the sessions in ${ }^{a} \mathrm{I}_{k}^{F}$, that is the sessions in ${ }^{a} \mathbf{I}_{k}^{F}$ preserve their position in $t$ - space ${ }^{\dagger}$. The "conditional exchange of weights" between sessions in ${ }^{a} \mathrm{I}_{k}$ and sessions in ${ }^{a} \mathrm{I}_{k}^{F}$ takes place continuously until one of the following happen:

(II.1.a) The backlog clearing time of sessions in ${ }^{a} I_{k}$ becomes equal to the backlog clearing time of sessions which empty their backlog $\mathrm{k}+1$-th in order under $\pi_{a}$ (sessions in ${ }^{a} \mathrm{I}_{k+1}$ ), or

(II.1.b) sessions in ${ }^{a} \mathrm{I}_{k}$ can not be compressed any further in $\phi$-space (their busy periods be uniformly expanded), because some session will miss its delay bound.

At the end of step (II.1) new $\phi$ 's will have been assigned to (all) streams in ${ }^{a} \mathbf{I}_{k}$ and ${ }^{a} I_{k}^{F}$ while streams in ${ }^{a} I_{k}^{P}$ will have maintained their original $\phi$ 's under $\pi_{a}$. Thus, a new policy $\pi_{b}$ is defined in terms of the new $\phi$ 's which is shown to be acceptable and more efficient than $\pi_{\alpha}$. At the end of step (II.1), the XMF process is applied to policy $\pi_{b}$ (modified $\pi_{a}$ ) from the beginning (from step (0))

(II.2) Else i f ${ }^{a} I_{k}$ is not compressible then a uniform expansion of the busy period of sessions in ${ }^{a} I_{k}$ is not feasible. In this case, ${ }^{a} \mathrm{I}_{k}$ is divided into two subsets, that is ${ }^{a} \mathrm{I}_{k}={ }^{a} \mathrm{I}_{k}^{C} \cup{ }^{a} \mathrm{I}_{k} \mathrm{NC}$, where: ${ }^{a} \mathrm{I}_{k}^{C}$ is the maximum subset of ${ }^{a} \mathrm{I}_{k}$ which is compressible in $\phi$ - space and ${ }^{a} \mathrm{I}_{k}^{N C}={ }^{a} \mathrm{I}_{k} \backslash{ }^{a} \mathrm{I}_{k}^{C}$.

(II.2.a) If ${ }^{a} \mathrm{I}_{k}^{C} \neq \emptyset$ then the two sets ${ }^{a} \mathrm{I}_{k}^{C}$ and ${ }^{a} \mathbf{I}_{k}^{N C}$ are separated by performing an infinitesimal uniform expansion of the busy periods of sessions in ${ }^{a} \mathbf{I}_{k}^{C}$ (at the same time the weights of sessions in ${ }^{a} \mathrm{I}_{k}^{F}$ are increased in such a way that they receive the same amount of work up to the end of the (modified) busy periods of sessions in ${ }^{a} \mathrm{I}_{k}^{C}$ as they did under $\pi_{a}$ (as at step (II.1))), resulting in a new policy $\pi_{b}$. After step (II.2.a), the XMF process is applied to policy $\pi_{b}$ (modified $\pi_{a}$ ) from the beginning (from step (0)).

(II.2.b) If ${ }^{a} \mathrm{I}_{k}^{C}=\emptyset$ then no stream in ${ }^{a} \mathrm{I}_{k}$ may be compressed in $\phi$ - space any further and the next set ${ }^{a} \mathrm{I}_{k+1}$ needs to be considered. Thus the XMF process continues from step (I).

(III) End of the XMF process. At this step the unique optimal policy $\pi o$ (see Proposition 3 ) has been determined, that is the original acceptable policy $\pi_{a}$ has been optimized. Under the resulting policy $\pi_{o}$ the QOS sensitive sessions are assigned some weights $\phi_{i}^{\pi_{o}}, i=1, \ldots, N$ and the best effort traffic is assigned weight $\phi_{b e}^{\pi_{o}}=1-\sum_{i=1}^{N} \phi_{i}^{\pi_{o}}$.

$\dagger$ see proof of Proposition 1 in the Appendix.

Fig. 2. Flowchart and description of XMF process.

From Propositions 1 and 2, it is easily concluded that: 1) $\pi_{o}$ is the only policy that remains unchanged under the XMF process; that is, $\forall \pi_{a} \in \Pi, \operatorname{XMF}\left(\pi_{a}\right)=\pi_{a} \Leftrightarrow \pi_{a}=\pi_{o}$ and 2) for any $\pi_{a} \in \Pi, \operatorname{XMF}\left(\pi_{a}\right)=\pi_{o}$ is more efficient than $\pi_{a}$, except for the case where $\pi_{a}=\pi_{o}$. In view of the above, the following proposition is self-evident.

Proposition 3: Policy $\pi_{o}, \pi_{o}=\operatorname{XMF}(\pi)$ for any $\pi \in \Pi$ is optimal and unique.

\section{B. Properties of the Optimal $\phi$ Assignment}

In this section, some properties of the optimal policy $\pi_{o}$ are provided. It is shown that in order to determine $\pi_{o}$ it is sufficient to observe the all-greedy system at certain time instants which coincide with either the delay bound or the backlog clearing 
time of some session. For this reason, the notion of the checkpoints is introduced.

Definition 3: Let $\tau_{0}=0$, that is, $\tau_{0}$ coincides with the beginning of the system busy period of the all-greedy system. Let $\left\{\tau_{m}\right\}_{m=1}^{M}, M \leq 2 N$ denote the ordered set of distinct time instants which coincide with either the delay bound or the backlog clearing time of some session. The time instant $\tau_{m}, m=0, \ldots, M$ will be referred to as the $m$ th ordered checkpoint.

Definition 4: Let $d_{i}=\left\{m: \tau_{m}=D_{i}\right\}$. That is, checkpoint $\tau_{d_{i}}$ coincides with the time instant at which the deadline of session $i$ expires. The following quantities are defined for session $i, i=1, \ldots, N$ at all checkpoints $\tau_{m}$ such that $D_{i} \leq \tau_{m}<e_{i}$

$$
\begin{aligned}
\phi_{i}^{-}\left(\tau_{m}\right) & =\frac{N_{i}\left(0, \tau_{m}\right)}{\hat{W}\left(0, \tau_{m}\right)} \\
\phi_{i}^{+}\left(\tau_{m}\right) & =\frac{\rho_{i}}{\hat{C}\left(\tau_{m}^{+}\right)}
\end{aligned}
$$

where $\hat{W}\left(0, \tau_{m}\right)=\sum_{k=1}^{m} \hat{W}\left(\tau_{k-1}, \tau_{k}\right)$.

The quantity $\phi_{i}^{-}\left(\tau_{m}\right)$ is expressed in terms of the values of the associated quantities (session's requirements, NBSA work) left of $\tau_{m}$ and refers to the evolution of the system for $t$ less than $\tau_{m}$. On the other hand, the quantity $\phi_{i}^{+}\left(\tau_{m}\right)$ is expressed in terms of the value of the NBSA bandwidth right of $\tau_{m}$.

The quantity $\phi_{i}^{-}\left(\tau_{m}\right)$ represents the fraction of the total NBSA work that must be assigned to session $i$ in order for session $i$ to be assigned work exactly equal to $N_{i}\left(0, \tau_{m}\right)$ up to time $\tau_{m}$, given that session $i$ has not emptied its backlog before time $\tau_{m}$. In particular, the denominator of the right-hand side of (8) is the total amount of NBSA work which is assigned to backlogged sessions up to time $\tau_{m}$, including sessions which cleared their backlog earlier and are no longer backlogged at time $\tau_{m}$. Each session $i$, which is still backlogged at $\tau_{m}$, gets a fraction of this work equal to $\phi_{i}$, that is, it is assigned work equal to $\phi_{i} \cdot \sum_{k=1}^{m} \hat{W}\left(\tau_{k-1}, \tau_{k}\right)$. The quantity $\phi_{i}^{+}\left(\tau_{m}\right)$ represents the fraction of the NBSA bandwidth just after $\tau_{m}$ that must be assigned to session $i$ in order for session $i$ to be served with a rate equal to $\rho_{i}$. It is easily seen that this is sufficient to ensure that its requirements are satisfied for $t>\tau_{m}$, if session $i$ is assigned work at least equal to $N_{i}\left(0, \tau_{m}\right)$ up to time $\tau_{m}$. The usefulness of these quantities follows from Proposition 5.

Proposition 4: Under $\pi_{o}, e_{i}>D_{i}, \forall i \in \operatorname{QoS}(\mathrm{QoS} \triangleq$ $\{1,2, \ldots, N\})$. That is, each QoS sensitive session empties its backlog after checkpoint $\tau_{d_{i}}=D_{i}$.

Proof: For $t<D_{i}$, session $i$ requirements have zero value and, thus, they do not impose a restriction on the expansion of session $i$ busy period. This means that if $e_{i}<D_{i}$ session $i$ would be compressible in $\phi$ space, thus $\pi_{o}$ would not remain unchanged under XMF. If $e_{i}=D_{i}$, since for $D_{i} \rho_{i} \neq$ $0 A_{i}\left(0, D_{i}\right)-N_{i}\left(0, D_{i}\right)>0$, it is implied that an infinitesimal decrease of session's weight would be possible (session $i$ would be compressible in $\phi$ space).

Proposition 5: Under $\pi_{o}$, QoS sensitive session $i$ is assigned weight

$$
\phi_{i}^{\pi_{o}}=\left\{\begin{array}{l}
\phi_{i}^{-}\left(\tau_{k}\right) \text { if } \exists k \text { such that } \\
k=\min \left\{m: \phi_{i}^{-}\left(\tau_{m}\right) \geq \phi_{i}^{+}\left(\tau_{m}\right)\right\} \\
\phi_{i}^{+}\left(\tau_{k}\right), \quad k=\max \left\{n: \tau_{n}<\infty\right\}, \text { otherwise. }
\end{array}\right.
$$

Proof: As proved in Section III-A2 the optimal policy for the BETA-GPS system $\left(\pi_{o}\right)$ is the unique acceptable policy that remains "unchanged" under the XMF process. This implies that the weight $\phi_{i}^{\pi_{o}}$ assigned to QoS sensitive session $i$ under $\pi_{o}$ is such that the following two conditions are fulfilled.

C1) QoS requirements of session $i$ are met. In particular, for an arbitrary time instant $t$ :

C1a) QoS requirements of session $i$ up to time $t$ are met;

C1b) QoS requirements of session $i \forall t^{\prime}>t$ are met;

C2) Session $i$ is not compressible in $\phi$ space.

Let $\tau_{f}$ denote the last checkpoint with finite value, that is, $\tau_{f}=$ $\max \left\{\tau_{m}: \tau_{m}<\infty\right\}$. In order for condition C1a) to hold for $t=\tau_{f}$ it is sufficient that

$$
\phi_{i}^{\pi_{o}} \geq \max _{\tau_{m} \leq \tau_{f}}\left\{\phi_{i}^{-}\left(\tau_{m}\right)\right\} .
$$

In particular, (11) implies that for two consecutive checkpoints $\tau_{j-1}$ and $\tau_{j}$ with finite value: 1$) W_{i}\left(0, \tau_{j-1}\right) \geq N_{i}\left(0, \tau_{j-1}\right)$ and 2) $W_{i}\left(0, \tau_{j}\right) \geq N_{i}\left(0, \tau_{j}\right)$. Since $N_{i}(0, t)$ and $W_{i}(0, t)$ are both linear in $\left.\left[\tau_{j-1}, \tau_{j}\right], 1\right)$ and 2) imply that $W_{i}(0, t) \geq$ $N_{i}(0, t), \forall t \in\left[\tau_{j-1}, \tau_{j}\right]$.

Now it is easily seen that

$$
\phi_{i}^{\pi_{o}}=\max \left\{\max _{\tau_{m} \leq \tau_{f}}\left\{\phi_{i}^{-}\left(\tau_{m}\right)\right\}, \phi_{i}^{+}\left(\tau_{f}\right)\right\}
$$

where the "external" max takes into account condition $\mathrm{C} 1 \mathrm{~b}$ ) (session's requirements must be met not only up to $\tau_{f}$ but also for $t \geq \tau_{f}$ ) and equality holds due to condition $\mathrm{C} 2$ ).

In order to proceed the following must be proved.

Claim 1: For two consecutive checkpoints $\tau_{j-1}$ and $\tau_{j}$ with finite value $\left(\tau_{j} \leq \tau_{f}\right) \phi_{i}^{+}\left(\tau_{j}\right) \leq \phi_{i}^{+}\left(\tau_{j-1}\right) .^{3}$

Claim 2: For two consecutive checkpoints $\tau_{j-1}$ and $\tau_{j}$ with finite value $\left(\tau_{j} \leq \tau_{f}\right), \phi_{i}^{-}\left(\tau_{j-1}\right)<\phi_{i}^{+}\left(\tau_{j-1}\right) \Rightarrow \phi_{i}^{-}\left(\tau_{j-1}\right)<$ $\phi_{i}^{-}\left(\tau_{j}\right) .^{4}$

Claim 1 implies that

$$
\phi_{i}^{+}\left(\tau_{f}\right)=\min _{\tau_{m} \leq \tau_{f}}\left\{\phi_{i}^{+}\left(\tau_{m}\right)\right\} .
$$

If $\exists k$ such that $k=\min \left\{m: \phi_{i}^{-}\left(\tau_{m}\right) \geq \phi_{i}^{+}\left(\tau_{m}\right)\right\}$ Claim 2 holds for every $\tau_{j-1}, \tau_{j}, \tau_{j} \leq \tau_{k}$ and implies that $\phi_{i}^{-}\left(\tau_{k}\right)=$ $\max _{\tau_{m} \leq \tau_{k}}\left\{\phi_{i}^{-}\left(\tau_{m}\right)\right\}$. Obviously, $\phi_{i}^{-}\left(\tau_{k}\right) \geq \phi_{i}^{+}\left(\tau_{k}\right)$ implies that $\phi_{i}^{-}\left(\tau_{k}\right) \geq \phi_{i}^{-}\left(\tau_{j}\right), k \leq j \leq f$, since $\phi_{i}^{-}\left(\tau_{k}\right)$ is sufficient to ensure that session's QoS requirements are met for $t>\tau_{k}$. So, $\phi_{i}^{-}\left(\tau_{k}\right)=\max _{\tau_{m} \leq \tau_{f}}\left\{\phi_{i}^{-}\left(\tau_{m}\right)\right\}$ and (12), in conjunction with

$$
\begin{aligned}
& { }^{3} \text { Proof: } \\
& \hat{C}\left(\tau_{j-1}\right) \leq \hat{C}\left(\tau_{j}\right) \Leftrightarrow \phi_{i}^{+}\left(\tau_{j-1}\right)=\frac{\rho_{i}}{\hat{C}\left(\tau_{j-1}\right)} \geq \frac{\rho_{i}}{\hat{C}\left(\tau_{j}\right)}=\phi_{i}^{+}\left(\tau_{j}\right) . \\
& \phi_{i}^{-}\left(\tau_{j-1}\right)<\phi_{i}^{+}\left(\tau_{j-1}\right) \Leftrightarrow \frac{N_{i}^{j-1}}{\mathcal{W}_{1}^{j-1}}<\frac{\rho_{i}}{\mathcal{C}_{j-1}^{+}} \\
& \Leftrightarrow N_{i}^{j-1} \mathcal{C}_{j-1}^{+} \tau_{j-1}^{j}+N_{i}^{j-1} \mathcal{W}_{1}^{j-1} \\
& <\tau_{j-1}^{j} \rho_{i} \mathcal{W}_{1}^{j-1}+N_{i}^{j-1} \mathcal{W}_{1}^{j-1} \\
& \Leftrightarrow N_{i}^{j-1}\left(\mathcal{C}_{j-1}^{+} \tau_{j-1}^{j}+\mathcal{W}_{1}^{j-1}\right) \\
& <\left(N_{i}^{j-1}+\tau_{j-1}^{j} \rho_{i}\right) \mathcal{W}_{1}^{j-1} \\
& \Leftrightarrow N_{i}^{j-1} \mathcal{W}_{1}^{j}<\left(N_{i}^{j-1}+\tau_{j-1}^{j} \rho_{i}\right) \mathcal{W}_{1}^{j-1} \\
& \Leftrightarrow N_{i}^{j-1} \mathcal{W}_{1}^{j}<N_{i}^{j} \mathcal{W}_{1}^{j-1} \\
& \Leftrightarrow \phi_{i}^{-}\left(\tau_{j-1}\right)<\phi_{i}^{-}\left(\tau_{j}\right)
\end{aligned}
$$

where

$$
\begin{aligned}
& N_{i}^{x}=N_{i}\left(0, \tau_{x}\right), \quad \tau_{x-1}^{x}=\tau_{x}-\tau_{x-1} \\
& \mathcal{C}_{x}^{+}=\hat{C}\left(\tau_{x}^{+}\right), \mathcal{W}_{1}^{x}=\sum_{k=1}^{x} \hat{W}\left(\tau_{k-1}, \tau_{k}\right)
\end{aligned}
$$


(13), implies that $\phi_{i}^{\pi_{o}}=\phi_{i}^{-}\left(\tau_{k}\right)$. This means that Proposition 5 holds.

If $\nexists k$ such that $k=\min \left\{m: \phi_{i}^{-}\left(\tau_{m}\right) \geq \phi_{i}^{+}\left(\tau_{m}\right)\right\}$ Claim 2 holds for every $\tau_{j-1}, \tau_{j}$ with $\tau_{j} \leq \tau_{f}$ and implies that $\phi_{i}^{-}\left(\tau_{f}\right)=$ $\max _{\tau_{m} \leq \tau_{f}}\left\{\phi_{i}^{-}\left(\tau_{m}\right)\right\}$. Since $\phi_{i}^{-}\left(\tau_{f}\right)<\phi_{i}^{+}\left(\tau_{f}\right)(12)$ implies that $\phi_{i}^{\pi_{o}}=\phi_{i}^{+}\left(\tau_{f}\right)$ and Proposition 5 holds.

Proposition 6: Assume that $\tau_{k}, \hat{\mathrm{C}}\left(\tau_{k}^{+}\right), \forall k \leq j-1$ are known. $\tau_{j}$ is given by

$$
\tau_{j}=\min \left(\min _{i \in N e x_{j-1}} D_{i}, \min _{i \in \operatorname{Phi}_{j-1}} \operatorname{vct}_{i}\left(\tau_{j-1}\right)\right)
$$

where $N e x_{j-1}$ is the set of sessions with delay bound greater than $\tau_{j-1}, P h i_{j-1}$ is the set of sessions for which $\exists k \leq j-1$ : $\phi_{i}^{-}\left(\tau_{k}\right) \geq \phi_{i}^{+}\left(\tau_{k}\right)$ and have not cleared their backlog up to time $\tau_{j-1}$, and

$$
\operatorname{vct}_{i}\left(\tau_{j-1}\right)=\tau_{j-1}+\frac{A_{i}\left(0, \tau_{j-1}\right)-W_{i}\left(0, \tau_{j-1}\right)}{\phi_{i}^{\pi_{o}} \cdot \hat{C}\left(\tau_{j-1}^{+}\right)-\rho_{i}}
$$

is the virtual clearing time of session $i$ at $\tau_{j-1}$, that is, the backlog clearing time of session $i$ assuming that no other session is going to empty its backlog before session $i$ does.

Proof: Let the QoS sensitive sessions be divided in the following disjoint sets at $\tau_{j-1}$.

1) Empty $_{j-1}$ contains sessions which have emptied their backlog at the current or a previous checkpoint, i.e., Empty $_{j-1}=\left\{i \in\right.$ QoS $\left.: e_{i} \leq \tau_{j-1}\right\}$.

2) $N e x_{j-1}$ : contains sessions with a delay bound greater than $\tau_{j-1}$, i.e., $N e x_{j-1}=\left\{i \in \operatorname{QoS}: D_{i}>\tau_{j-1}\right\}$.

3) $P h i_{j-1}$ contains sessions for which $\exists k \leq j-1$ : $\phi_{i}^{-}\left(\tau_{k}\right) \geq \phi_{i}^{+}\left(\tau_{k}\right)$ and have not cleared their backlog up to time $\tau_{j}$.

4) Trans $_{j-1}$ contains sessions for which $\exists k \leq j-1$ : $\phi_{i}^{-}\left(\tau_{k}\right) \geq \phi_{i}^{+}\left(\tau_{k}\right)$ and have $D_{i} \leq \tau_{j-1}$.

According to the definition of the checkpoints, $\tau_{j}$ coincides with either the delay bound or the backlog clearing time of some session. Obviously, no future checkpoint is associated with sessions $i \in$ Empty $_{j-1}$, since $e_{i} \leq \tau_{j-1}$ and according to Proposition 4, $D_{i}<e_{i} \forall i \in$ Empty $_{j-1}$.

According to Proposition 4, all sessions $i \in N e x_{j-1}$ have $e_{i}>\min _{i \in N e x_{j-1}} D_{i}$. It is implied that if $\tau_{j}$ is associated with a session in $N e x_{j-1}$ it will have to be the session $k, k: D_{k}=$ $\min _{i \in N e x_{j-1}} D_{i}$. This "justifies" the first term of equation.

Claim 3: For two consecutive checkpoints $\tau_{j-1}$ and $\tau_{j}$ with finite value $\left(\tau_{j} \leq \tau_{f}\right), \phi_{i}^{-}\left(\tau_{j-1}\right)<\phi_{i}^{+}\left(\tau_{j-1}\right) \Rightarrow \phi_{i}^{+}\left(\tau_{j-1}\right)>$ $\phi_{i}^{-}\left(\tau_{j}\right){ }^{6}$

From Proposition 5, in conjunction with Claim 3, it is easily concluded that sessions in Trans $_{j-1}$ are assigned weight less

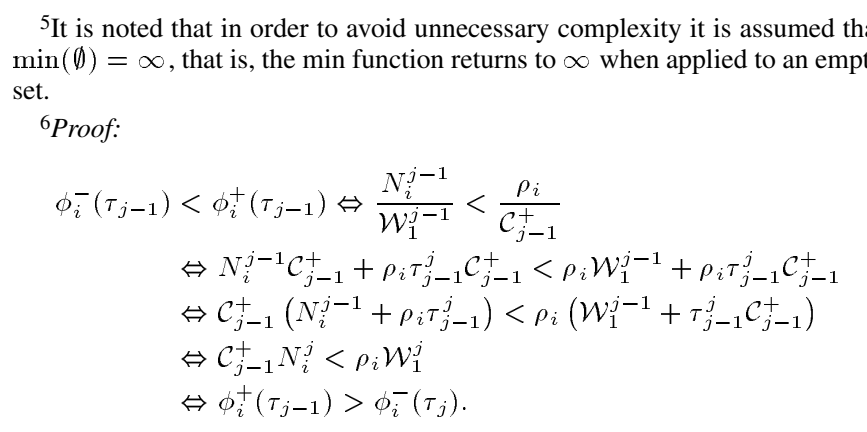

The notations are the same as in proof of Claim 2. than or equal to $\phi_{i}^{+}\left(\tau_{j-1}\right)$ under $\pi_{o}$, i.e., they are served with a rate less than $\rho_{i}$ in the interval $\left(\tau_{j-1}, \tau_{j}\right]$. This means that the slope of their service curve is less than the slope of their (greedy) arrival curve. Both curves are linear in $\left(\tau_{j-1}, \tau_{j}\right]$ and it is obvious that these two lines cannot cross each other, i.e., sessions in Trans $_{j-1}$ do not empty their backlog at $\tau_{j}$.

All sessions in $P h i_{j-1}$ are served with a rate greater than or equal to $\rho_{i}$ for $t>\tau_{j-1}$ so they could empty their backlog at $\tau_{j}$. For every session $i \in P h i_{j-1}$ the line with slope $\phi_{i}^{\pi_{\circ}} \hat{C}^{+}\left(\tau_{j-1}\right)$ which passes from the point $\left(\tau_{j-1}, W_{i}\left(0, \tau_{j-1}\right)\right.$ represents $W_{i}(0, t)$ for $t \geq \tau_{j-1}$ and has the form $y_{1}(t)=\phi_{i}^{\pi_{o}} \hat{C}^{+}\left(\tau_{j-1}\right)\left(t-\tau_{j-1}\right)+W_{i}\left(0, \tau_{j-1}\right)$. The line $y_{2}(t)=\sigma_{i}+\rho_{i} t$ represents the arrivals of the greedy session. These two lines cross each other at $t=$ $\left(\sigma_{i}+\phi_{i}^{\pi_{o}} \hat{C}^{+}\left(\tau_{j-1}\right) \tau_{j-1}-W_{i}\left(0, \tau_{j-1}\right)\right) /\left(\phi_{i}^{\pi_{o}} \hat{C}^{+}\left(\tau_{j-1}\right)-\rho_{i}\right)$ $=\tau_{j-1}+\left(A_{i}\left(0, \tau_{j-1}\right)-W_{i}\left(0, \tau_{j-1}\right)\right) /\left(\phi_{i}^{\pi_{o}} \cdot \hat{C}^{+}\left(\tau_{j-1}\right)-\rho_{i}\right)$ $=\operatorname{vct}_{i}\left(\tau_{j-1}\right)$. It is obvious that the only sessions for which their virtual clearing time represents their real backlog clearing time are the sessions whose virtual clearing time is equal to the minimum virtual clearing time.

\section{Optimal CAC Algorithm}

The CAC algorithm presented in this section determines progressively the optimal policy $\pi_{o}$, based on Propositions 5 and 6. It includes two conceptually distinct functions; one which (based on Proposition 5) examines whether the optimal weights of the QoS sensitive sessions can be determined at a specific checkpoint and another which (based on Proposition 6) determines the next checkpoint.

Assume that all the checkpoints $\left(\tau_{k}\right)$ and the corresponding values of the NBSA bandwidth $\left(\hat{\mathrm{C}}\left(\tau_{k}^{+}\right)\right)$were known. Proposition 5 could be used in order to determine the optimal weights of the QoS sensitive sessions. A sequential consideration of all the checkpoints would be required since, according to (the first case of) Proposition 5, for each QoS sensitive session $i$ the first checkpoint $\tau_{j}$ at which the condition $\phi_{i}^{-}\left(\tau_{j}\right) \geq \phi_{i}^{+}\left(\tau_{j}\right)$ would hold should be determined in order to determine the optimal weight of session $i$; if $\tau_{m}$ denotes this particular checkpoint for session $i$ the optimal weight of session $i$ is $\phi_{i}^{\pi_{o}}=$ $\phi_{i}^{-}\left(\tau_{m}\right)$. The weights of the QoS sensitive sessions not fulfilling the aforementioned condition at any checkpoint (with a finite value) would be determined at the last checkpoint with a finite value, as prescribed by the second case of Proposition 5; if $\tau_{m}$ denotes the last checkpoint with a finite value checkpoint, the optimal weights of such sessions $i$ are $\phi_{i}^{\pi_{o}}=\phi_{i}^{+}\left(\tau_{m}\right)$.

In the sequel, it is said that session $i$ is examined at $\tau_{j}$, when it is checked whether the condition $\phi_{i}^{-}\left(\tau_{j}\right) \geq \phi_{i}^{+}\left(\tau_{j}\right)$ (in the first case of Proposition 5), referred to as the condition, holds for session $i$ at $\tau_{j}$. The interval $\left[D_{i}, e_{i}\right)$ is referred to as the examination interval of session $i$; notice that the quantities $\phi_{i}^{-}\left(\tau_{j}\right), \phi_{i}^{+}\left(\tau_{j}\right)$ used in the condition are defined for session $i$ at $\tau_{j}$ only if $D_{i} \leq \tau_{j}<e_{i}$ (see Definition 4). In addition, it is said that session $i$ is assigned an appropriate weight, when session $i$ meets the condition at $\tau_{j}$ and is assigned the optimal weight $\phi_{i}^{\pi_{o}}=\phi_{i}^{-}\left(\tau_{j}\right)$.

The algorithm presented in this section employs Proposition 5 in a similar way as that described above in order to determine the optimal weights of the QoS sensitive sessions. That is, the checkpoints are considered sequentially and each session $i$ is 
Determine $\pi_{o}\left(C_{G}, Q o S\right)$

A. $j=0$, Trans $_{0}=P h i_{0}=$ Empt $_{0}=\emptyset$, Nex $x_{0}=Q o S, \tau_{0}=0$

B. repeat (B1.-B6.) until $\left(\phi_{i}=\phi_{i}^{\pi_{o}} \quad \forall i \in N e x_{0}\right)$

B1. $j=j+1, \tau_{j}=\min \left\{\min _{i \in N e x_{j-1}} D_{i}, \min _{i \in \text { Phi }_{j-1}} v c t_{i}\left(\tau_{j-1}\right)\right\}$

B2. If $\left(\tau_{j}=\infty\right)$ then

$\left\{\forall i \in\right.$ Trans $_{j-1}$

$\left\{\phi_{i}=\phi_{i}^{\pi_{o}}=\phi_{i}^{+}\left(\tau_{j-1}\right)\right.$, if $\sum_{i \in Q_{o S}} \phi_{i} \geq 1$ then error $\}$ goto $C$.

\}

B3. $P E_{j}=\left\{i \in P h i_{j-1}: \operatorname{vct}_{i}\left(\tau_{j-1}\right)=\tau_{j}\right\}$, Empty $_{j}=$ Empty $_{j-1} \cup P E_{j}$

B4. $T P_{j}=\left\{i \in\right.$ Trans $\left._{j-1}: \phi_{i}^{-}\left(\tau_{j}\right) \geq \phi_{i}^{+}\left(\tau_{j}\right)\right\}$ $N P_{j}=\left\{i \in N e x_{j-1}: D_{i}=\tau_{j}, \phi_{i}^{-}\left(\tau_{j}\right) \geq \phi_{i}^{+}\left(\tau_{j}\right)\right\}$ $N T_{j}=\left\{i \in N e x_{j-1}: D_{i}=\tau_{j}, \phi_{i}^{-}\left(\tau_{j}\right)<\phi_{i}^{+}\left(\tau_{j}\right)\right\}$

B5. $N e x_{j}=N e x_{j-1} \backslash\left(N P_{j} \cup N T_{j}\right)$, Trans $_{j}=\left(\right.$ Trans $\left._{j-1} \backslash T P_{j}\right) \cup N T_{j}$

B6. $\forall i \in N P_{j} \cup T P_{j}$

$\left\{\phi_{i}=\phi_{i}^{\pi_{o}}=\phi_{i}^{-}\left(\tau_{j}\right)\right.$, if $\sum_{i \in Q_{o S}} \phi_{i} \geq 1$ then error $\}$ $P h i_{j}=\left(P h i_{j-1} \backslash P E_{j}\right) \cup N P_{j} \cup T P_{j}$

C. $\phi_{b e}=1-\sum_{i \in Q O S} \phi_{i}$

Fig. 3. Pseudocode of optimal CAC algorithm.

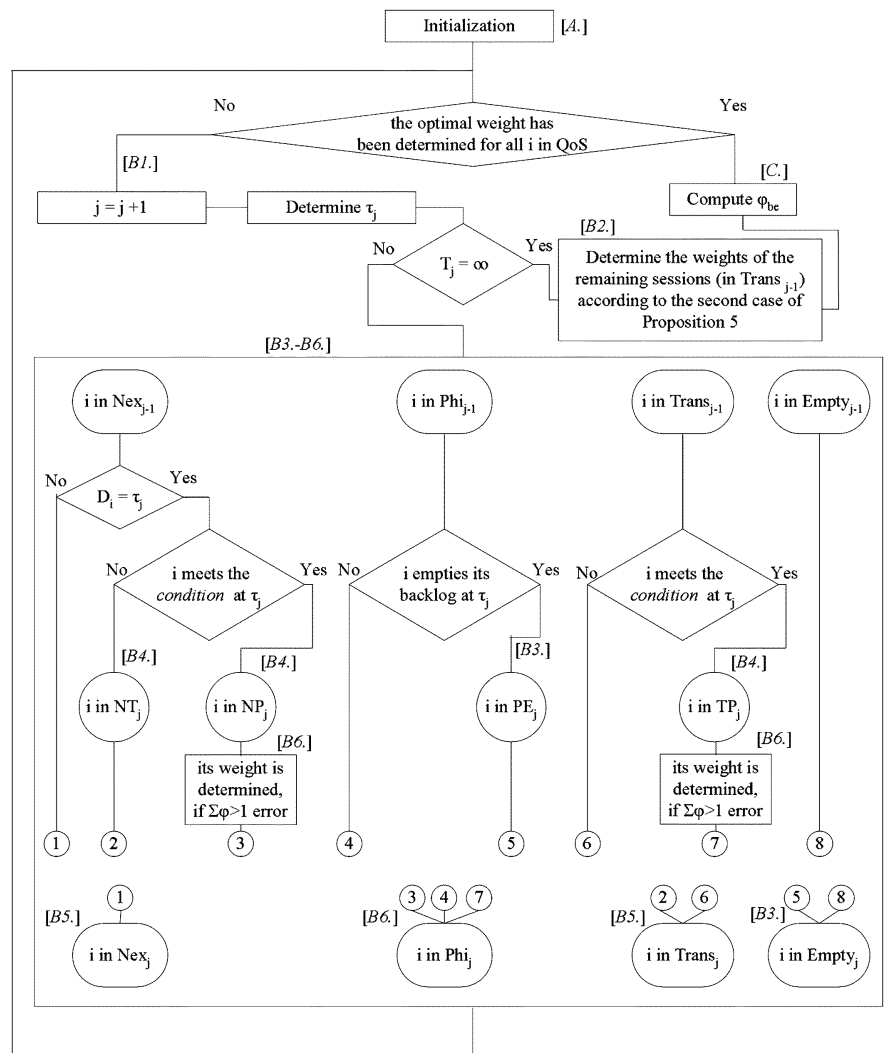

Fig. 4. Flowchart of the optimal CAC algorithm. Steps of the pseudocode at which the corresponding actions take place are illustrated within square brackets.

examined at the checkpoints that are within the examination interval of session $i$. At the checkpoint that the condition is met for session $i$ for the first time, session $i$ is assigned an appropriate weight. At the last checkpoint with a finite value, sessions that have not been assigned a weight are assigned a weight equal to that prescribed by the second case of Proposition 5. However, in the framework of the algorithm the checkpoints $\left(\tau_{k}\right)$ and the corresponding values of the NBSA bandwidth $\left(\hat{\mathrm{C}}\left(\tau_{k}^{+}\right)\right)$are determined on the fly (by using Proposition 6) since they are not $a$ priori known; only the first checkpoint $\left(\tau_{0}=0\right)$ and the initial value of the NBSA bandwidth $\left(\hat{\mathrm{C}}\left(\tau_{0}^{+}\right)=C_{G}\right)$ are known.

The pseudocode of the algorithm is provided in Fig. 3, while Fig. 4 illustrates the flowchart of the algorithm. $C_{G}$ is the bandwidth controlled by the GPS scheduler and QoS is the set of
QoS sensitive sessions under investigation. The algorithm iterates over the checkpoints starting at $\tau_{0}=0$; index $j$ runs over the checkpoints, while index $i$ runs over the QoS sensitive sessions. At each iteration a checkpoint $\left(\tau_{j}\right)$ is considered, and each QoS sensitive session $i$ belongs to one of the following disjoint sets $\mathrm{Phi}_{j}, \mathrm{Empty}_{j}, \mathrm{Nex}_{j}, \mathrm{Trans}_{j}$. These are the same sets of sessions as those defined in the proof of Proposition 6. However, in the framework of the algorithm, their conceptual interpretation is slightly different. More specifically:

- Set $P h i_{j}$ contains sessions that fulfill the condition at $\tau_{j}$ or some previous checkpoint and have not emptied their backlog yet (up to $\tau_{j}$ ). In the framework of the algorithm these are the sessions whose weights have been determined (since, according to the algorithm, when the condition is met for a session at a checkpoint this session is assigned an appropriate weight).

- Set Empty $y_{j}$ contains sessions which have emptied their backlog at (or before) $\tau_{j}$. Their weights are known, since (as explained in Step B3) sessions that empty their backlog at $\tau_{j}$ belong in $P h i_{k}$ for some $0<k \leq j-1$, that is, their weights have been determined at some previous checkpoint.

- Set $N e x_{j}$ contains sessions whose delay bound is less than $\tau_{j}$. In the framework of the algorithm, this set is also referred to as the set of not examined sessions, since these sessions need not be examined at $\tau_{j} ; \tau_{j}$ is not within the examination interval of any session in $N_{e x}$, thus the condition is definitely not met by any of them at $\tau_{j}$.

- Set Trans $s_{j}$ contains the rest of the sessions, that is, sessions that were examined at a previous checkpoint but their weights were not determined.

Next, a detailed description of the algorithm is provided. In the description it is assumed that all the checkpoints up to $\tau_{j-1}$ are known, as well as the sets of sessions Phi $_{k}$, Empty $_{k}$, Nex $_{k}$, Trans $_{k}$, for $k \leq j-1$ and the weights of the sessions in $P h i_{j-1}$ and in Empty ${ }_{j-1}$ (these weights are determined during previous iterations). Notice that this holds for $j=1$, since $\tau_{0}=0$ and $C\left(\tau_{0}^{+}\right)=C_{G}$; at $\tau_{0}$ all QoS sensitive sessions belong to $N e x_{0}$ (since their delay bounds are greater than $\tau_{0}=0$ ), while all other sets are empty.

A) Initialization of the algorithm. Initially, the checkpoint which corresponds to the beginning of the system busy period of the all-greedy system (that is, $\tau_{0}=0$ ) is considered. At $\tau_{0}$, Steps B2)-B6) of the algorithm are not executed, since the sets of sessions are already known (as explained at the beginning, all sets are empty except $N e x_{0}$ ).

B) Main loop B1)-B6) is executed until the weights of all QoS sensitive sessions are determined.

B1) Using Proposition 6, the value of time at the current checkpoint $\tau_{j}$ is computed. The weights of sessions in $P h i_{j-1}$, which are needed in order to determine $\tau_{j}$, have been determined using Proposition 5 at some previous checkpoint, since $P h i_{j-1}$ contains sessions which fulfill the condition at some previous checkpoint. Thus, the quantities needed to determine the next checkpoint are known or can be computed.

B2) If $\tau_{j}=\infty$, the previous checkpoint $\left(\tau_{j-1}\right)$ was the one with the maximum finite value of time, and according to 
the second case of Proposition 5 the weights of the sessions whose weight has not been determined yet must be determined at $\tau_{j-1}$. Thus, sessions $i$ in $\operatorname{Trans}_{j-1}$ are assigned a weight equal to $\phi_{i}^{+}\left(\tau_{j-1}\right)$. Such sessions (whose optimal weight is determined according to the second case of Proposition 5) have a backlog clearing time which tends to infinity. If the sum of the weights assigned to QoS sensitive sessions is greater or equal to one, then the sessions are not schedulable and the algorithm terminates. ${ }^{7}$

B3) Sessions which empty their backlog at the current check-

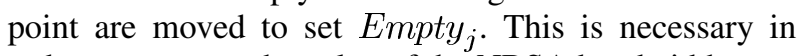
order to compute the value of the NBSA bandwidth at $\tau_{j}$ $\left(\hat{C}\left(\tau_{j}^{+}\right)\right)$; according to (5), the set of sessions that empty their backlog up to $\tau_{j}$ and their weights must be known.

As shown in the Proof of Proposition 6, only sessions in $P h i_{j-1}$ may empty their backlog at $\tau_{j}$, and from the sessions in $P h i_{j-1}$ those whose virtual clearing time at $\tau_{j-1}$ are equal to $\tau_{j}$ empty their backlog at $\tau_{j}$. Thus, set $\mathrm{PE}_{j} \subseteq P h i_{j-1}$ corresponds to the set of sessions which has been examined at a previous checkpoint; the weights have been determined at a previous checkpoint and empty their backlog at $\tau_{j}$.

B4) As mentioned, the algorithm uses Proposition 5 in order to determine the optimal weights of the QoS sensitive sessions. According to Proposition 5, if $\tau_{j}$ is the first checkpoint, within the examination interval of session $i$, at which the condition is met for session $i$ (that is, $\phi_{i}^{-}\left(\tau_{j}\right) \geq \phi_{i}^{+}\left(\tau_{j}\right)$ holds), then the optimal weight for session $i$ is $\phi_{i}^{\pi_{o}}=\phi_{i}^{-}\left(\tau_{j}\right)$. At this step, the algorithm checks whether the condition is met for two kinds of sessions.

- Sessions which have already been examined at some previous checkpoint but did not fulfill the condition (that is, sessions in $\operatorname{Trans}_{j-1}$ ). Set $T P_{j} \subseteq$ Trans $_{j-1}$ contains the sessions that fulfill the condition for the first time at $\tau_{j} . T P_{j}$ is moved into set $P h i_{j}$ at Step B6); the rest of the sessions in Trans $_{j-1}$ remain in set Trans $_{j}$.

- Sessions which are examined for the first time at $\tau_{j}$ (sessions for which $\tau_{j}=D_{i}$, that is, $\tau_{j}$ is the beginning of their examination interval). From these sessions, those fulfilling the condition form the set $N P_{j} \subseteq$ $N e x_{j-1}$ (sessions which are examined for the first time at $\tau_{j}$ and their weights can be determined at $\tau_{j}$ since they meet the condition at $\tau_{j}$ ), while the rest of these sessions form the set $N T_{j} \subseteq N e x_{j-1}$ (sessions which are examined for the first time at the current checkpoint $\left(\tau_{j}\right)$ but their weights cannot determined at $\tau_{j}$ ). At Steps B5) and B6) the main sets are properly updated.

B5) The sets $N e x_{j}$ and Trans $_{j}$ are determined by properly updating the corresponding sets at $\tau_{j-1}$. More specifically, all sessions in $N e x_{j-1}$ that are examined at $\tau_{j}$ (sets $N P_{j}$ and $N T_{j}$ ) are removed from $N e x_{j-1}$; sessions in Trans $_{j-1}$ that fulfill the condition at $\tau_{j}\left(\right.$ set $\left.T P_{j}\right)$ are removed from $\operatorname{Trans}_{j-1}$, while sessions in $N e x_{j-1}$ that are examined at $\tau_{j}$ and do not meet the condition at $\tau_{j}$ (set $N T_{j}$ ) are added to $\operatorname{Trans}_{j-1}$.

\footnotetext{
${ }^{7} \mathrm{All} \phi \mathrm{s}$ are considered to be initially undefined. $\sum_{i \in \mathrm{QoS}} \phi_{i}$ denotes the summation over all sessions that have been assigned a weight by the algorithm.
}

B6) At this step, the weight assignment for the sessions whose weight is determined at $\tau_{j}$ is performed. If the sum of the weights assigned to QoS sensitive sessions is greater than or equal to one, then the sessions are not schedulable and the algorithm terminates. In addition, set $P h i_{j}$ is determined by removing from $P h i_{j-1}$ the sessions that empty their backlog at $\tau_{j}\left(\right.$ set $\left.\mathrm{PE}_{j}\right)$ and by adding the sessions whose weights are determined at $\tau_{j}$ (sets $N P_{j}$ and $T P_{j}$ ).

C) The weight of the best effort traffic session $\left(\phi_{\text {be }}^{\pi_{o}}\right)$ is computed.

\section{PuRe QoS SysteM}

In a system where only QoS sensitive sessions are present, the existence of an extra session, denoted as "dummy," may be assumed and the presented algorithm be applied. The modified algorithm for the pure QoS system is referred to as modified optimal CAC algorithm (MOCA).

The MOCA is exactly the same as the optimal CAC algorithm for the BETA-GPS system except the check of the sum of the weights assigned to QoS sensitive sessions at steps B2) and B6), which should be replaced by ... "if $\left(\sum_{i \in \mathrm{QoS}} \phi_{i}>1\right.$ or $\phi_{i}=$ 0 ), then error"..., since the "dummy" session can be assigned a weight equal to zero. In addition, at Step C) $\phi_{\text {be }}$ should be replaced by $\phi_{d}, d$ for "dummy."

The input of the MOCA is a traffic mix consisting only of QoS sensitive sessions $i, i=1, \ldots, N$. If the MOCA finds a solution, it returns a $\phi$ assignment $\left(\phi_{1}, \phi_{2}, \ldots, \phi_{N}, \phi_{d}\right)$. Obviously, the QoS sensitive sessions can be admitted being assigned weights $\left(\phi_{1}, \phi_{2}, \ldots, \phi_{N}\right)$ (or normalized to sum to one $\left.\left(\phi_{1}, \phi_{2}, \ldots, \phi_{N}\right) \cdot\left(1-\phi_{d}\right)^{-1}\right)$. If the MOCA does not find a solution, then this implies that a solution does not exist, since the MOCA minimizes $\sum_{i=1}^{N} \phi_{i}$. In this sense, the MOCA can be considered as optimal for the pure QoS system.

\section{A. Minimal Bandwidth Requirement}

Another capability that could be required by a CAC scheme for the pure QoS system is to be able to compute the minimum capacity of the GPS server $C_{G(\min )}$ required to support the $N$ QoS sensitive sessions (and the appropriate $\phi$ assignment). (The capacity not used by the GPS server could be used by another server. The scheme under which such a partitioning could be realized is out of the scope of this paper.) In this case, the following proposition is useful.

Proposition 7: Suppose that an acceptable policy is computed by the MOCA in a pure QoS system where the GPS scheduler controls capacity $C_{G}$. QoS sensitive sessions, assigned the computed weights and served by a GPS scheduler controlling capacity $C_{G}\left(1-\phi_{d}\right)$, do meet their QoS requirements.

Proof: Assume that an acceptable policy $\left(\phi_{1}, \phi_{2}, \ldots\right.$, $\left.\phi_{N}, \phi_{d}\right)$ has been computed by the MOCA. If $\phi_{d}=0$, the proposition holds. If $\phi_{d} \neq 0$, any $\left(\sigma_{d}, \rho_{d}, D_{d}\right)$ can be chosen to describe the "dummy" session as long as it does conform to the assumption that the "dummy" session empties its backlog last in order and to the stability condition $\left(\sum_{i \in \mathrm{QoS} \cup d} \rho_{i} \leq 1\right)$. Let $\rho_{d}=\phi_{d} C_{G}$, and let $\sigma_{d}$ and $D_{d}$ be arbitrary but properly selected so that the "dummy" session empties its backlog last in order.

Since the greedy system corresponds to the worst case arrival scenario resulting in the maximum delay experienced by the 
QoS sensitive sessions, their requirements are satisfied under any other arrival scenario including the following: Consider the "dummy" session not starting greedy at the beginning of the system busy period but with $\sigma_{d}=0$ and transmitting with a constant rate equal to $\rho_{d}=\phi_{d}$. $C_{G}$. It is easily seen that this scenario is equivalent to a pure QoS system where the GPS server controls capacity equal to $C_{G}\left(1-\phi_{d}\right)$.

Proposition 7 states that the QoS sensitive sessions would meet their requirements even in a more stringent environment, where the capacity controlled by the GPS server is equal to $C_{G}\left(1-\phi_{d}\right)$. This indicates that if it is desirable to compute the minimum capacity of the GPS server $\left(C_{G(\min )}\right)$ required to support the $N$ QoS sensitive sessions, the following recursive process can be followed, which amounts to cutting slices of size $\phi_{d(n)} C_{n}$ from the capacity $C_{n}$ (with $C_{1}=C_{G}$ ) controlled by the GPS scheduler at the $n$th iteration until the last slice becomes smaller than an arbitrary predefined small quantity $\epsilon$.

$$
\begin{aligned}
& \text { compute } C_{G(\min )}\left(\operatorname{Nex} x_{0}, C_{G}\right) \\
& n=1, C_{(1)}=C_{G} \\
& \text { for }(; ;) \\
& \left\{\phi_{d(n)}=\phi_{d}\left(\operatorname{MOCA}\left(\operatorname{Nex} x_{0}, C_{(n)}\right)\right)\right. \\
& C_{(n+1)}=C_{(n)}\left(1-\phi_{d(n)}\right) \\
& \text { if }\left(\phi_{d(n)} C_{(n)}<\epsilon\right), \text { then }\left\{C_{G(\min )}=C_{(n+1)}, \text { exit }\right\} \\
& n=n+1\} .
\end{aligned}
$$

$N e x_{0}$ is the traffic mix (consisting only of QoS sensitive sessions) and $\epsilon$ is an arbitrary small positive number. $\phi_{d(n)}$ is the weight assigned to the "dummy" session by the MOCA, assuming that the GPS scheduler controls capacity $C_{(n)}$. The process stops when $\phi_{d(n)} C_{(n)}$ becomes less than a predefined quantity $\epsilon$.

The feasibility of this process and the fact that it can approximate as closely as desired the minimum GPS capacity required to support the $N$ QoS sensitive sessions can be easily concluded. In particular, let $\phi_{d(1)}$ be the weight assigned to the "dummy" session at the first iteration of the process. The weight assigned to the "dummy" session is the weight not needed by the QoS sensitive sessions. If $\phi_{d(1)}=0$, then the optimal solution is determined, since the MOCA minimizes $\sum_{i=1}^{N} \phi_{i}$. If $\phi_{d(1)} \neq 0$, then, according to Proposition 7, bandwidth $\left(1-\phi_{d(1)}\right) C_{G}$ is sufficient to satisfy QoS sensitive sessions requirements. Applying MOCA at the second iteration leads to the computation of the maximum $\phi$ not needed by the QoS sensitive sessions for the more stringent system $\left(\phi_{d(2)}, \phi_{d(2)} \leq \phi_{d(1)}\right) .{ }^{8}$ If $\phi_{d(2)}=0$, the optimal solution is determined, else the process is repeated. Due to the fact that $\phi_{d(n)}$ becomes smaller at each iteration, the aforementioned process approximates as closely as desired the solution to the problem of minimization of the required capacity to admit the QoS sensitive sessions.

\footnotetext{
${ }^{8}$ It is easy to prove that $\phi_{d(2)} \leq \phi_{d(1)}$ by contradiction. In particular, suppose that $\phi_{d(2)}>\phi_{d(1)}$. Since QoS sensitive sessions meet their delay bounds under $\left(\phi_{1(2)}, \ldots, \phi_{N(2)}\right), \sum_{i=1}^{N} \phi_{i(2)}=1-\phi_{d(2)}$, when the GPS server controls capacity $C_{G}\left(1-\phi_{d(1)}\right)$ they will not miss their delay bound when the GPS server controls capacity $C_{G}$, that is, the policy $\left(\phi_{1(2)}, \ldots, \phi_{N(2)}\right)$ is an acceptable policy for the case where the GPS server controls capacity $C_{G}$. This implies that $\left(\phi_{1(1)}, \ldots, \phi_{N(1)}\right), \sum_{i=1}^{N} \phi_{i(1)}=1-\phi_{d(1)}$ computed by MOCA at the first iteration was not the policy with the minimum $\sum_{i=1}^{N} \phi_{i}$ (contradiction).
}

\section{DisCUSSION AND NUMERICAL RESUlTS}

\section{A. Relation to the Effective Bandwidth-Based CAC}

Deterministic effective bandwidth [8] can be used in a straightforward way to give a simple and elegant CAC scheme. A similar approach is followed in [4] for the deterministic part of their analysis. The deterministic effective bandwidth of a $\left(\sigma_{i}, \rho_{i}, D_{i}\right)$ session is given by $w_{i}^{\text {eff }}=\max \left\{\rho_{i},\left(\sigma_{i}\right) /\left(D_{i}\right)\right\}$. It is easy to see that the requirements of the QoS sensitive sessions are satisfied if they are assigned weights such that $\phi_{i} C_{G}=w_{i}^{\text {eff }}\left(\left(\phi_{i}\right) /\left(\phi_{j}\right)=\left(w_{i}^{\text {eff }}\right) /\left(w_{j}^{\text {eff }}\right), \forall i, j \in\right.$ QoS $)$. In this section, the presented algorithm is compared with the effective bandwidth-based CAC scheme. The effective bandwidth-based CAC is tighter than the CAC, which is based on rate proportional weighting $\left(\left(\phi_{i} / \phi_{j}\right)=\left(\rho_{i} / \rho_{j}\right)\right)$, which is used for comparison in [3] (see also [10]).

In addition, it is noted that the effective bandwidth-based CAC scheme can be considered as a special case of the presented algorithm. If the presented algorithm is denied the ability to "remember" which sessions and when they empty their backlog, the NBSA bandwidth, as computed by the algorithm, has a constant value which is equal to $C_{G}$ and each checkpoint coincides with some session's delay bound. It is easily seen that in this special case the algorithm would assign to session $i$ weight equal to $\max \left\{\phi_{i}^{-}\left(\tau_{d_{i}}\right), \phi_{i}^{+}\left(\tau_{d_{i}}\right)\right\}$, since the algorithm would be completely unable to keep track of the evolution of the greedy system. This implies ${ }^{9}$ that $\phi_{i} C_{G}=\max \left\{\rho_{i},\left(\sigma_{i}\right) /(D i)\right\}=$ $w_{i}^{\text {eff }}$, that is, the algorithm would assign to each session the same weight as the effective bandwidth-based CAC would.

\section{B. Graphical Interpretation}

In this section, an intuitive examination of the algorithm is attempted. The examination is based on the graphical representation of the mean values of the associated quantities.

Since the GPS scheduler is work conserving, $C_{G} \cdot t=$ $\sum_{i \in T F} W_{i}(0, t)$, where $T F=$ QoS $\cup$ be in the best effort aware system and $T F=$ QoS in the pure QoS system, holds for arbitrary $t$ in the system busy period. This implies that

$$
C_{G}=\sum_{i \in T F} \bar{W}_{i}(0, t)
$$

where $\bar{W}_{i}(0, t)=W_{i}(0, t) / t$ is the mean work assigned to session $i$ in $(0, t]$. The mean requirements of session $i$ in the interval $(0, t]$ are

$$
\bar{N}_{i}(0, t)= \begin{cases}\frac{\sigma_{i}+\rho_{i}\left(t-D_{i}\right)}{t}, & t \geq D_{i} \\ 0, & t<D_{i}\end{cases}
$$

which implies

$$
\partial \bar{N}_{i}(0, t) / \partial t=\left(\rho_{i} D_{i}-\sigma_{i}\right) / t^{2}, \quad t \geq D_{i} .
$$

Although session $i$ requirements are always an increasing function of time (for $t \geq D_{i}$ ), the mean requirements of a session are an increasing (decreasing) function of time if $\rho_{i} D_{i}>$ $\sigma_{i}\left(\rho_{i} D_{i}<\sigma_{i}\right)$.

${ }^{9}$ From Definition 4 , it is concluded that in this special case (where the NBSA bandwidth is considered equal to $\left.C_{G}\right) \phi_{i}^{-}\left(\tau_{d_{i}}\right)=\left(\sigma_{i}\right) /\left(C_{G} D_{i}\right)$ and $\phi_{i}^{+}\left(\tau_{d_{i}}\right)=\left(\rho_{i}\right) /\left(C_{G}\right)$ for all QoS sensitive sessions. 


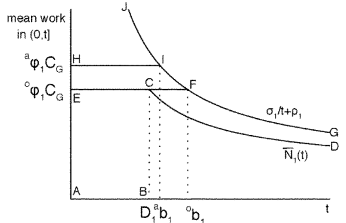

(I.)
(II.)

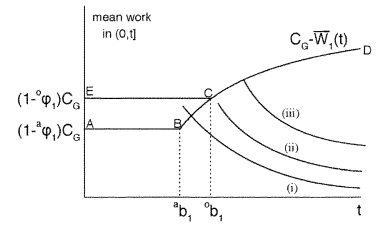

Fig. 5. (I) Mean service received by the session which empties its backlog first in order under the optimal policy $\pi_{o}$ and under the policy $\pi_{a}$. (II) Mean work assigned to other sessions under the optimal policy $\pi_{o}$ and under some other policy $\pi_{a}$.

Condition $W_{i}(0, t) \geq N_{i}(0, t), t \geq D_{i}$, which must hold in order for QoS sensitive session $i$ requirements to be satisfied, implies that $\bar{W}_{i}(0, t) \geq \bar{N}_{i}(0, t), t \geq D_{i}$. For $t \geq e_{i}$, where $e_{i}$ is the backlog clearing time of session $i$, session $i$ is served with a constant rate (equal to $\rho_{i}$ ). Thus

$$
\bar{W}_{i}(0, t)=\frac{\sigma_{i}+\rho_{i} t}{t}=\frac{\sigma_{i}}{t}+\rho_{i}, \quad t \geq e_{i}
$$

In Fig. 5(I), the mean service received by the QoS sensitive session which empties its backlog first in order (it is assumed that it is only one for simplicity and will be referred to as session 1) under the optimal policy $\pi_{o}$ (curve EFG) and under some other policy, $\pi_{a}$ (curve HIG), is shown.

$\bar{W}_{1}(0, t)$ is constant (linear parts EF and HI for policies $\pi_{o}$ and $\pi_{a}$, respectively) as long as the session is backlogged. According to (19), the service curve of the session coincides with the curve $\left(\sigma_{1}\right) /(t)+\rho_{1}$ for $t \geq e_{1}=\mathrm{b}_{1}$. This implies that the backlog clearing time of the session corresponds to point F under $\pi_{o}$ and I under $\pi_{a}$. From Fig. 5(I), it is evident that session's requirements (curve ABCD) are satisfied under both policies.

In Fig. 5(II), the quantity $C_{G}-\bar{W}_{1}(0, t)$ (curve ABCD under $\pi_{a}$, curve ECD under $\pi_{o}$ ), which represents the mean work available to other sessions, is shown. In this figure the following observations can be made.

1) Under $\pi_{o}$ the requirements of a session with mean requirements of the form (i) can be satisfied, while under $\pi_{a}$ they cannot.

2) Under both policies, a session with mean requirements of the form (ii) can be admitted. In addition, under both policies the best effort traffic is assigned the same fraction of $C_{G}-$ $\bar{W}_{1}(t)$ for $t>^{o} \mathrm{~b}_{1}$, but this corresponds to a greater weight under $\pi_{o}$. The former observations illustrated the optimality of the presented algorithm.

3) It may also be observed that under both policies a session with mean requirements of the form (iii) can be admitted. In this example, the minimum required server capacity has the same value under both policies and $\sum_{i=1}^{2} \phi_{i}=1$. This illustrates that the optimal solution for the pure QoS system is, in general, not unique, since there exist several policies under which $\sum_{i=1}^{N} \phi_{i}$ is minimized.

Finally, it should be mentioned that no session with a deterministic effective bandwidth greater than that corresponding to the straight line $A B \ldots$ in Fig. 5(II) would be admitted along with session 1 [whose deterministic effective bandwidth corresponds to the straight line $E C F \ldots$ in Fig. 5(I)].
TABLE I

SESSIONS UNDER INVESTIGATION

\begin{tabular}{|c|c|c|c|c|}
\hline Case 1 & $s_{1}$ & $s_{2}$ & & $s_{3}$ \\
\hline Case 2 & $s_{1}$ & & $s_{2}$ & $s_{3}$ \\
\hline$\sigma_{i}$ & 0.04 & 0.16 & 0.04 & 0.64 \\
$\rho_{i}$ & 0.01 & 0.01 & 0.04 & 0.01 \\
$D_{i}$ & 1 & 4 & 4 & 16 \\
$w_{i}^{\text {eff }}$ & 0.04 & 0.04 & 0.04 & 0.04 \\
\hline
\end{tabular}
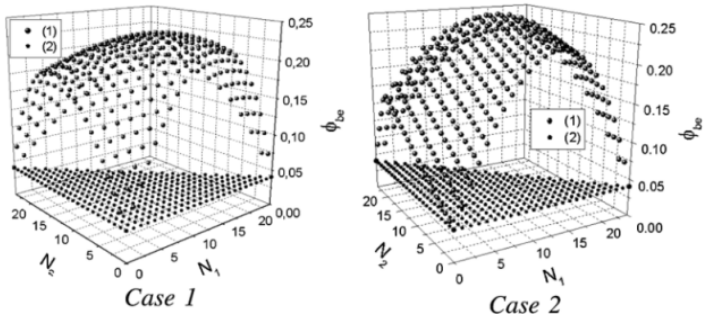

Fig. 6. Weight assigned to the best effort traffic according to the (1) optimal BETA-GPS CAC and (2) effective bandwidth-based CAC scheme, both under the constraint $N_{1}+N_{2}+N_{3}=24$. Minimum guaranteed rate to the best effort traffic is $\phi_{\mathrm{be}} C_{G}$.

\section{Numerical Results}

In this section, some numerical results are presented for the BETA-GPS system. Although the algorithm can support an arbitrary number of delay classes the numerical investigation is limited to the case of three delay classes. Two cases are investigated.

Case 1:The traffic mix consists only of QoS sensitive sessions whose mean requirements are a decreasing function of time for $t \geq D_{i}, \forall i$. The sessions under investigation for this case are shown in Table I. All quantities are considered normalized with respect to the link capacity $\mathrm{C}$.

In order to compare the presented algorithm with the effective bandwidth-based CAC scheme, the following scenario is considered. The effective bandwidth-based CAC scheme admits the maximum number of sessions under the constraint that a nonzero weight remains to be assigned to best effort traffic. From Table I it can be seen that the effective bandwidth of each QoS sensitive session is $1 / 25$ of the server's capacity (which is considered to be equal to the link capacity $\left(C_{G}=C\right)$ ), implying that for the BETA-GPS system at most 24 QoS sensitive sessions can be admitted under the effective bandwidth-based CAC scheme. This means that $N_{1}+N_{2}+N_{3}=24$ must hold and that the best effort traffic is assigned weight equal to 0.04 for each such triplets $\left(N_{1}, N_{2}\right.$, and $N_{3}$ denote the number of admitted sessions of type $s_{1}, s_{2}$, and $s_{3}$, respectively). For each triplet $\left(N_{1}, N_{2}, N_{3}\right), N_{1}+N_{2}+N_{3}=24$, the weight assigned to the best effort traffic by the optimal BETA-GPS CAC scheme is computed. The results are illustrated in Fig. 6.

It is easily seen that the improvement achieved by the optimal algorithm depends on the diversity of the traffic mix. For heterogeneous traffic mixes a significant improvement is achieved. On the other hand, for pure homogeneous traffic mixes (only one type of session) the optimal algorithm cannot result in any improvement.

Case 2:The traffic mix consists of both types of QoS sensitive sessions (with increasing and decreasing mean requirements). To demonstrate this case session $s_{2}$ is replaced by a session with the same effective bandwidth but with mean requirements 
which are an increasing function of time (see Table I). The same scenario as in Case 1 is followed. The results are illustrated in Fig. 6. The achieved improvement is less than in Case 1, in particular when sessions of type $s_{1}$ are a minor part of the traffic mix.

The above results suggest that the presented algorithm for the BETA-GPS system may achieve a significant improvement, compared to the effective bandwidth-based CAC scheme when the traffic mix is heterogeneous and consists of bursty sessions, ${ }^{10}$ that is, sessions whose mean requirements are a decreasing function of time. It is noted that the case where the traffic mix consists of QoS sensitive session whose mean requirements are an increasing function of time for $t \geq D_{i}, \forall i$ is not considered, since in this special case the problem of CAC is trivial; that is, the stability condition is sufficient to ensure that the QoS sensitive sessions do not violate their delay bounds, and obviously the described optimal CAC algorithm does not improve the resource utilization.

\section{Complexity of the Algorithm}

Consider the following simplistic implementation of the algorithm, which is in direct correspondence with the pseudocode of the algorithm. The state of the QoS sensitive sessions is kept in a $N \times 6$ matrix. Each row of the matrix corresponds to a QoS sensitive session $i \sim\left(\sigma_{i}, \rho_{i}, D_{i}\right)$ and includes the following fields: $\sigma_{i}, \rho_{i}, D_{i}, \phi_{i}, \operatorname{vct}_{i}\left(\tau_{j-1}\right), \operatorname{set}_{i}\left(\tau_{j-1}\right), \operatorname{set}_{i}\left(\tau_{j}\right)$, where $\phi_{i}$ is the weight assigned to session $i, \operatorname{vct}_{i}\left(\tau_{j-1}\right)$ is the virtual clearing time of session $i$ at the $j-1$ iteration and $\operatorname{set}_{i}\left(\tau_{j-1}\right)$ $\left(\operatorname{set}_{i}\left(\tau_{j}\right)\right)$ indicates the set that session $i$ belongs to at the $j-$ $1(j)$ iteration. In addition, assume that the values of the quantities $\tau_{j}, \hat{C}\left(\tau_{j}^{+}\right), \hat{W}\left(0, \tau_{j}\right), \sum_{i \in \mathrm{QoS}} \phi_{i}$ for each checkpoint are stored.

Initially, the sessions are sorted with respect to delay in ascending order, incurring a complexity of $O(N \log N)$. At each iteration of the algorithm a checkpoint is examined. It can easily be verified that each step of each iteration is of complexity $\mathrm{O}(\mathrm{N})$. More specifically, computing the quantities $\phi_{i}^{-}\left(\tau_{j}\right), \phi_{i}^{+}\left(\tau_{j}\right), \operatorname{vct}_{i}\left(\tau_{j-1}\right)$ for a session $i$ can be done in $\mathrm{O}(1)$ and, thus, computing these quantities for all sessions examined at an iteration can be done in $\mathrm{O}(\mathrm{N})$. The update of a set corresponds to properly adjusting the values of $\operatorname{set}_{i}\left(\tau_{j-1}\right)$ and $\operatorname{set}_{i}\left(\tau_{j}\right)$ and is of complexity $\mathrm{O}(\mathrm{N})$. Finding the minimums at Step B1) and the update of $\hat{C}\left(\tau_{j}^{+}\right)$[which can also take place during Step B3)] are of complexity $\mathrm{O}(\mathrm{N})$, while the update of $\hat{W}\left(0, \tau_{j}\right)$ is an $\mathrm{O}(1)$ operation. Thus, the update of the system at each checkpoint is performed in $O(N)$ complexity. There are at most $2 N$ checkpoints, implying that the computational complexity of the algorithm is $O\left(N^{2}\right)$.

\section{CONCLUSION}

In this paper, the problem of allocating optimal weights to sessions being served according to the GPS scheduling disci-

\footnotetext{
${ }^{10}$ At this point it should be noted that sessions are characterized as "burtsy" and "nonbursty" from the perspective of the required service rate (and not from the perspective of the arrival rate); as a threshold the mean rate of the session is used. More specifically, the service rate of a session whose mean requirements are a decreasing function of time must exceed (for some time interval) its mean rate in order for the QoS requirements of the sessions to be met and, thus, some kind of burstiness is expected to occur in the session's service rate; such sessions are characterized as bursty.
}

pline at a single node has been addressed. The derivation of the optimal solution is based on a procedure capable of transforming any acceptable weight allocation to the optimal one; based on this procedure, a CAC algorithm that computes the optimal weight assignment directly has been derived.

Since the proposed CAC scheme is optimal, it bounds the resource utilization that can be achieved by a GPS scheduler at a single node and, thus, helps in comparing the efficiency of other simpler CAC schemes and the comparison of GPS with other scheduling disciplines. For example, it can be used to investigate how much worse GPS is as compared to earliest deadline first (EDF), which is known to be the optimal scheduler for the single-node case.

Apart from its value as a performance bound, whether the $O\left(N^{2}\right)$ optimal algorithm retains enough gain to be used in practice in place of $O(N)$ deterministic effective bandwidthbased algorithm remains to be investigated.

In addition, it should be noted that in order to be able to sustain an optimal performance in a dynamic environment (new sessions arriving, other ending) the algorithm has to be re-executed upon any change in the traffic mix in order to recompute the optimal weights. This requirement emphasizes an inherent drawback of the GPS scheduling discipline, which is that it cannot fully exploit the available bandwidth under a static allocation of weights.

\section{APPENDIX}

\section{A. Proof of Proposition 1 (Section III.A)}

Due to space limitations, the following notations are used in the proof of Proposition 1:

$$
\begin{aligned}
{ }_{x}^{y} \mathcal{P}_{z}^{w} & =\sum_{i \in{ }^{y} \mathbf{I}_{z}^{w}}{ }^{x} \phi_{i}, \quad{ }_{x}^{y} \mathcal{F}_{z}^{w} \\
& =\sum_{i \in{ }^{y} \mathbf{I}_{z}^{w}}{ }^{x} \mathrm{f}_{i}, \quad{ }^{y} \mathcal{R}_{z}^{w}=\sum_{i \in{ }^{y} \mathbf{I}_{z}^{w}} \rho_{i} \\
\overline{{ }_{x}^{y} \mathcal{P}_{z}^{w}} & =1-{ }_{x}^{y} \mathcal{P}_{z}^{w}, \quad \overline{{ }_{x}^{y} \mathcal{P}_{z}^{w}+{ }_{p}^{q} \mathcal{P}_{s}^{r}}=1-{ }_{x}^{y} \mathcal{P}_{z}^{w}-{ }_{p}^{q} \mathcal{P}_{s}^{r} \\
\overline{{ }^{y} \mathcal{R}_{z}^{w}} & =C_{G}-{ }^{y} \mathcal{R}_{z}^{w}, \\
\bar{y} \mathcal{R}_{z}^{w}+{ }^{q} \mathcal{R}_{s}^{r} & =C_{G}-{ }^{y} \mathcal{R}_{z}^{w}-{ }^{q} \mathcal{R}_{s}^{r} \\
{ }_{x}^{y} \mathcal{C}_{z}^{w} & ={ }^{y} \hat{\mathrm{C}}\left({ }^{x} \mathrm{~b}_{z}^{w}\right), \quad l_{w, k}^{z, x}={ }^{z} \mathrm{~b}_{w}-{ }^{x} \mathrm{~b}_{k} \\
{ }^{y} \hat{W}_{z, w}^{x, k} & ={ }^{y} \hat{W}\left({ }^{z} \mathrm{~b}_{w},{ }^{x} \mathrm{~b}_{k}\right), \\
{ }_{x}^{y} \mathcal{W}_{z}^{w} & =\sum_{m=z}{ }^{y} \hat{W}_{x, m-1}^{x, m} .
\end{aligned}
$$

Only the proof for Steps II.1a) and II.1b) is provided. The proof for Step II.2.a) is similar.

First, it is proved that the "conditional exchange of weights" between sessions in ${ }^{a} \mathrm{I}_{k}$ and sessions in ${ }^{a} \mathrm{I}_{k}^{F}$ is feasible, that is, the total decrease of the weights of sessions in ${ }^{a} \mathrm{I}_{k}$ is sufficient to balance the necessary total increase of the weights of sessions in ${ }^{a} \mathrm{I}_{k}^{F}$ so that the condition [see II.1)] is met. It is also shown that the best effort traffic is assigned a greater weight under $\pi_{b}$, that is $\pi_{b}$ is more efficient than $\pi_{a}$. Then, it is shown that the QoS sensitive sessions do not miss their delay bound under $\pi_{b}$.

Assume that the described "conditional exchange of weights" between sessions in ${ }^{a} \mathrm{I}_{k}$ and sessions in ${ }^{a} \mathrm{I}_{k}^{F}$ is feasible. Let ${ }^{a} \phi_{i}$ denote the weight assigned to session $i$ under $\pi_{a}$. Under $\pi_{b}$, 
sessions in ${ }^{a} \mathrm{I}_{k}^{P}$ preserve their position in $\phi$ space, sessions in ${ }^{a} \mathrm{I}_{k}$ are compressed in $\phi$ space, and sessions in ${ }^{a} \mathrm{I}_{k}^{F}$ are decompressed in $\phi$ space, i.e.

$$
\begin{aligned}
{ }^{b} \phi_{i} & ={ }^{a} \phi_{i}, \quad i \in{ }^{a} \mathrm{I}_{k}^{P} ; \quad{ }^{b} \phi_{i}={ }^{a} \phi_{i}-{ }^{b} \mathrm{f}_{i}, \quad i \in{ }^{a} \mathrm{I}_{k} \\
{ }^{b} \phi_{i} & ={ }^{a} \phi_{i}+{ }^{b} \mathrm{f}_{i}, \quad i \in{ }^{a} \mathrm{I}_{k}^{F} ; \quad{ }^{b} \mathrm{f}_{i}>0 \quad \forall i \in \operatorname{QoS} .
\end{aligned}
$$

1) Sessions in ${ }^{a} \mathrm{I}_{k} \cup{ }^{a} \mathrm{I}_{k}^{F}$ Are Backlogged at ${ }^{a} \mathrm{~b}_{k-1}$ Under $\pi_{b}$ : If Step II.1a) is followed, ${ }^{b} \mathrm{I}_{k}={ }^{a} \mathrm{I}_{k} \cup{ }^{a} \mathrm{I}_{k+1}$ and ${ }^{b} \mathrm{~b}_{k}>{ }^{a} \mathrm{~b}_{k}$ holds for the (intermediate) policy $\pi_{b}$. If Step II.1b) is followed, ${ }^{a} \mathrm{I}_{k}={ }^{b} \mathrm{I}_{k}$ but ${ }^{b} \mathrm{~b}_{k}>{ }^{a} \mathrm{~b}_{k}$. In both cases, sessions in ${ }^{a} \mathrm{I}_{k}$ empty their backlog at ${ }^{b} \mathrm{~b}_{k}$ under $\pi_{b},{ }^{b} \mathrm{~b}_{k}>{ }^{a} \mathrm{~b}_{k}$ and sessions in ${ }^{a} \mathrm{I}_{k}^{F}$ are assigned the same amount of work under $\pi_{b}$ and under $\pi_{a}$ over the interval $\left(0,{ }^{b} \mathrm{~b}_{k}\right]$. This means that since sessions in ${ }^{a} \mathrm{I}_{k} \cup{ }^{a} \mathrm{I}_{k}^{F}$ are still backlogged at time ${ }^{a} \mathrm{~b}_{k-1}$ under $\pi_{a}$, they are backlogged at time ${ }^{a} \mathrm{~b}_{k-1}$ (and $\forall t<{ }^{a} \mathrm{~b}_{k-1}$ ) under $\pi_{b}$ too.

2) Sessions in ${ }^{a} \mathrm{I}_{k}^{P}$ Preserve Their Position in $t$ Space: Sessions in ${ }^{a} I_{k}^{P}$ preserve their position in $\phi$ space (they are assigned the same weights under $\pi_{a}$ and $\pi_{b}$ ), and all sessions in ${ }^{a} \mathrm{I}_{k} \cup{ }^{a} \mathrm{I}_{k}^{F}$ (and the best effort traffic) empty their backlog for $t \geq{ }^{a} \mathrm{~b}_{k}>{ }^{a} \mathrm{~b}_{k-1}$, under $\pi_{a}$ and under $\pi_{b}$, by assumption. Obviously, ${ }^{a} \mathrm{~b}_{0}={ }^{b} \mathrm{~b}_{0}=0$ and ${ }^{b} \hat{\mathrm{C}}\left({ }^{a} \mathrm{~b}_{0}^{+}\right)={ }^{a} \hat{\mathrm{C}}\left({ }^{a} \mathrm{~b}_{0}^{+}\right)=C_{G}$. Since ${ }^{b} \phi_{i}={ }^{a} \phi_{i}, \forall i \in{ }^{a} \mathrm{I}_{k}^{P}$, it is implied that ${ }^{b} \mathrm{~b}_{1}={ }^{a} \mathrm{~b}_{1},{ }^{b} \mathrm{I}_{1}={ }^{a} \mathrm{I}_{1}$, and ${ }^{b} \hat{\mathrm{C}}\left({ }^{a} \mathrm{~b}_{1}^{+}\right)={ }^{a} \hat{\mathrm{C}}\left({ }^{a} \mathrm{~b}_{1}^{+}\right)$. Similarly, it is concluded that

$$
{ }^{b} \mathrm{~b}_{m}={ }^{a} \mathrm{~b}_{m} \wedge{ }^{b} \mathrm{I}_{m}={ }^{a} \mathrm{I}_{m}, \quad m=0, \ldots, k-1
$$

and

$$
{ }_{b}^{b} \mathcal{C}_{m}^{+}={ }_{a}^{a} \mathcal{C}_{m}^{+}, \quad m=0, \ldots, k-1 .
$$

This means that in both cases [Step II.1.a) or II.1.b)] sessions in ${ }^{a} \mathrm{I}_{k}^{P}$ preserve their position in $t$ space.

3) Sessions in ${ }^{a} I_{k}^{P}$ Preserve Their Position int Space: CASE A: ${ }^{a} \mathrm{~b}_{k+1}<\infty$. Sessions in ${ }^{a} \mathrm{I}_{k}$ empty their backlog at ${ }^{a} \mathrm{~b}_{k}$ under $\pi_{a}$. This implies $W_{i}\left(0,{ }^{a} \mathrm{~b}_{k}\right)=A_{i}\left(0,{ }^{a} \mathrm{~b}_{k}\right) \forall i \in{ }^{a} \mathrm{I}_{k}$, i.e.,

$$
{ }^{a} \phi_{i}{ }_{a}^{a} \mathcal{W}_{1}^{k}=\sigma_{i}+\rho_{i}{ }^{a} \mathrm{~b}_{k}, \quad i \in{ }^{a} \mathrm{I}_{k} .
$$

Sessions in ${ }^{b} \mathrm{I}_{k}$ empty their backlog at ${ }^{b} \mathrm{~b}_{k}$ under $\pi_{b}$, i.e.,

$$
{ }^{b} \phi_{i b}^{b} \mathcal{W}_{1}^{k}=\sigma_{i}+\rho_{i}{ }^{b} \mathrm{~b}_{k}, \quad i \in{ }^{b} \mathrm{I}_{k} .
$$

In both cases [Steps (II.1a) and (II.1b)] under examination ${ }^{a} \mathrm{I}_{k} \subseteq{ }^{b} \mathrm{I}_{k}$, implying that (24) holds $\forall i \in{ }^{a} \mathrm{I}_{k}$. Subtracting (23) from (24) implies

$$
\text { Cond. } 1:{ }^{b} \phi_{i b}^{b} \mathcal{W}_{1}^{k}-{ }^{a} \phi_{i}{ }_{a}^{a} \mathcal{W}_{1}^{k}=\rho_{i} l_{k, k}^{a, b}, \quad i \in{ }^{a} \mathrm{I}_{k} .
$$

Sessions in ${ }^{a} \mathbf{I}_{k}^{F}$ are assigned the same amount of work up to time ${ }^{b} \mathrm{~b}_{k}$ under $\pi_{a}$ and under $\pi_{b}$, i.e.,

$$
\text { Cond. } 2:{ }^{b} \phi_{i b}^{b} \mathcal{W}_{1}^{k}={ }^{a} \phi_{i}\left({ }_{a}^{a} \mathcal{W}_{1}^{k}+{ }^{a} \hat{\mathrm{W}}_{a, k}^{b, k}\right), \quad i \in{ }^{a} \mathrm{I}_{k}^{F} \text {. }
$$

From (21) and (22), it is implied that

$$
{ }^{b} \hat{\mathrm{W}}_{b, m-1}^{b, m}={ }^{a} \hat{\mathrm{W}}_{a, m-1}^{a, m}, \quad m=1, \ldots, k-1 .
$$

Using (22)

$$
{ }^{b} \hat{\mathrm{W}}_{b, k-1}^{b, k}={ }_{a}^{a} \mathcal{C}_{k-1}^{+} l_{k, k}^{a, b}+{ }^{a} \hat{\mathrm{W}}_{a, k-1}^{a, k} .
$$

Equations (27) and (28) imply that

$$
{ }_{b}^{b} \mathcal{W}_{1}^{k}={ }_{a}^{a} \mathcal{W}_{1}^{k}+{ }_{a}^{a} \mathcal{C}_{k-1}^{+} l_{k, k}^{a, b} .
$$

In addition, it is obvious that

$$
{ }^{a} \hat{\mathrm{W}}_{a, k}^{b, k}={ }_{a}^{a} \mathcal{C}_{k}^{+} l_{k, k}^{a, b} .
$$

Conditions 1 and 2, using (29) and (30), become

${ }^{b} \mathrm{f}_{i}\left({ }_{a}^{a} \mathcal{W}_{1}^{k}+{ }_{a}^{a} \mathcal{C}_{k-1}^{+} l_{k, k}^{a, b}\right)=\left({ }^{a} \phi_{i a}^{a} \mathcal{C}_{k-1}^{+}-\rho_{i}\right) l_{k, k}^{a, b}, \quad i \in{ }^{a} \mathrm{I}_{k}$

${ }^{b} \mathrm{f}_{i}\left({ }_{a}^{a} \mathcal{W}_{1}^{k}+{ }_{a}^{a} \mathcal{C}_{k-1}^{+} l_{k, k}^{a, b}\right)={ }^{a} \phi_{i}\left({ }_{a}^{a} \mathcal{C}_{k}^{+}-{ }_{a}^{a} \mathcal{C}_{k-1}^{+}\right) l_{k, k}^{a, b}, \quad i \in{ }^{a} \mathrm{I}_{k}^{F}$

Equations (31) and (32) provide the necessary relations to determine the ${ }^{b} f_{i}$ s under the assumption that the "conditional exchange of weights" is feasible. In order to prove that the assumption holds, it is sufficient to show that the total decrease of the weights of sessions in ${ }^{a} \mathrm{I}_{k}$ is sufficient to balance the total increase of the weights of sessions in ${ }^{a} \mathrm{I}_{k}^{F}$.

4) Decrease of the Weights of Sessions in ${ }^{a} \mathbf{I}_{k}$ Is Greater Than the Increase of the Weights of Sessions in ${ }^{a} \mathrm{I}_{k}^{F}$ : Summing over all $i \in{ }^{a} \mathrm{I}_{k}$, (31) gives

$$
{ }_{b}^{a} \mathcal{F}_{k}\left({ }_{a}^{a} \mathcal{W}_{1}^{k}+{ }_{a}^{a} \mathcal{C}_{k-1}^{+} l l_{k, k}^{a, b}\right)=\left({ }_{a}^{a} \mathcal{P}_{k a}^{a} \mathcal{C}_{k-1}^{+}-{ }^{a} \mathcal{R}_{k}\right) l_{k, k}^{a, b} .
$$

After some algebra, the following equality can be verified

$$
{ }_{a}^{a} \mathcal{P}_{k a}^{a} \mathcal{C}_{k-1}^{+}-{ }^{a} \mathcal{R}_{k}=\left({ }_{a}^{a} \mathcal{C}_{k}^{+}-{ }_{a}^{a} \mathcal{C}_{k-1}^{+}\right)\left(1-{ }_{a}^{a} \mathcal{P}_{k}-{ }_{a}^{a} \mathcal{P}_{k}^{P}\right)
$$

Dividing (32) by (33), due to (34), results in

$$
{ }^{b} \mathrm{f}_{i}={ }^{a} \phi_{i b}{ }_{i b} \mathcal{F}_{k}\left(1-{ }_{a}^{a} \mathcal{P}_{k}-{ }_{a}^{a} \mathcal{P}_{k}^{P}\right)^{-1}, \quad i \in{ }^{a} \mathrm{I}_{k}^{F} .
$$

Equation (35) is the main result of this section. Summing over all sessions in ${ }^{a} \mathrm{I}_{k}^{F}$ (and using $\sum_{i \in \mathrm{QoS}}{ }^{a} \phi_{i}+{ }^{a} \phi_{\mathrm{be}}=1$ )

$$
{ }_{b}^{a} \mathcal{F}_{k}^{F}=a^{a} \mathcal{P}_{k}^{F}\left({ }^{a} \phi_{\mathrm{be}}+{ }_{a}^{a} \mathcal{P}_{k}^{F}\right)^{-1}{ }_{b}^{a} \mathcal{F}_{k} \Leftrightarrow{ }_{b}^{a} \mathcal{F}_{k}^{F}<{ }_{b}^{a} \mathcal{F}_{k} .
$$

This means that the total decrease of the weights of sessions in ${ }^{a} \mathrm{I}_{k}$ is greater than the total increase of the weights of sessions in ${ }^{a} \mathrm{I}_{k}^{F}$. So, the described "conditional exchange of weights" is feasible. In addition, the best effort traffic is assigned a greater weight under the intermediate policy $\pi_{b}$

$$
\begin{aligned}
{ }^{b} \phi_{\text {be }} & =1-\sum_{i \in \mathrm{QoS}}{ }^{b} \phi_{i}=1-{ }_{b}^{b} \mathcal{P}_{k}^{P}-{ }_{b}^{b} \mathcal{P}_{k}-{ }_{b}^{b} \mathcal{P}_{k}^{F} \\
& ={ }_{a}^{a} \overline{\mathcal{P}}_{k}^{P}-{ }_{a}^{a} \mathcal{P}_{k}+{ }_{b}^{a} \mathcal{F}_{k}-{ }_{a}^{a} \mathcal{P}_{k}^{F}-{ }_{b}^{a} \mathcal{F}_{k}^{F} \\
& ={ }^{a} \phi_{\text {be }}-{ }_{b}^{a} \mathcal{F}_{k}^{F}+{ }_{b}^{a} \mathcal{F}_{k}>{ }^{a} \phi_{\mathrm{be}} .
\end{aligned}
$$


Equation (35) implies that

$$
{ }^{b} \mathrm{f}_{i}\left({ }^{b} \mathrm{f}_{j}\right)^{-1}={ }^{a} \phi_{i}\left({ }^{a} \phi_{j}\right)^{-1}, \quad i, j \in{ }^{a} \mathbf{I}_{k}^{F} .
$$

Under $\pi_{a}$ and $\pi_{b}$, the same sessions empty their backlog in $\left(0,{ }^{b} \mathrm{~b}_{k}\right]$ (implying that $\left.{ }^{b} \hat{\mathrm{C}}\left({ }^{b} \mathrm{~b}_{k}^{+}\right)={ }^{a} \hat{\mathrm{C}}\left({ }^{b} \mathrm{~b}_{k}^{+}\right)\right)$and sessions in ${ }^{a} \mathrm{I}_{k}^{F}$ receive, by assumption, the same amount of work over $\left(0,{ }^{b} \mathrm{~b}_{k}\right]$ under both policies. In conjunction with (38) it can be concluded that sessions in ${ }^{a}{ }_{k}^{F}$ preserve their position in $t$ space under $\pi_{b}$. It is easy to show that this holds for the best effort traffic too.

CASE B: ${ }^{a} \mathrm{~b}_{k+1}=\infty$. This case is examined only for Step II.1.a) $\left({ }^{b} \mathrm{I}_{k}={ }^{a} \mathrm{I}_{k} \cup{ }^{a} \mathrm{I}_{k+1}\right.$ and $\left.{ }^{b} \mathrm{~b}_{k}>{ }^{a} \mathrm{~b}_{k}\right)$, since the proof for Step II.1.b) is exactly the same as in CASE A.

5) Equation (35) Holds for CASE B Too: In Case B (and assuming that Step II.1.a) was followed) the following equations hold under $\pi_{b}$ and $\pi_{a}$, since ${ }^{b} \mathrm{~b}_{k}=\infty$ and ${ }^{a} \mathrm{~b}_{k+1}=\infty$ :

$$
\begin{aligned}
{ }^{b} \phi_{i b}{ }^{b} \mathcal{C}_{k-1}^{+} & =\rho_{i} \quad i \in{ }^{a} \mathrm{I}_{k} \cup{ }^{a} \mathrm{I}_{k}^{F} \\
{ }^{a} \phi_{i a}{ }^{a} \mathcal{C}_{k}^{+} & =\rho_{i} \quad i \in{ }^{a} \mathrm{I}_{k}^{F} .
\end{aligned}
$$

From (21) and (22)

$$
{ }^{a} \mathrm{~b}_{k-1}={ }^{b} \mathrm{~b}_{k-1} \wedge{ }_{a}^{a} \mathcal{C}_{k-1}^{+}={ }_{b}^{b} \mathcal{C}_{k-1}^{+} .
$$

Subtracting (39) from (40) implies

$$
{ }^{a} \phi_{i}\left({ }_{a}^{a} \mathcal{C}_{k}^{+}-{ }_{a}^{a} \mathcal{C}_{k-1}^{+}\right)={ }^{b} \mathrm{f}_{i a}^{a} \mathcal{C}_{k-1}^{+} \quad i \in{ }^{a} \mathrm{I}_{k}^{F} .
$$

Summing over all $i \in{ }^{a} \mathrm{I}_{k}$, (39) gives

$$
{ }_{b}^{a} \mathcal{F}_{k a}^{a} \mathcal{C}_{k-1}^{+}={ }_{a}^{a} \mathcal{P}_{k a}{ }_{a} \mathcal{C}_{k-1}^{+}-{ }^{a} \mathcal{R}_{k} .
$$

Dividing (42) by (43), due to (34), results in (35).

6) $\pi_{b}$ Is Acceptable: It has been shown that the "conditional exchange of weights" between sessions in ${ }^{a} \mathrm{I}_{k}$ and sessions in ${ }^{a} \mathrm{I}_{k}^{F}$ is feasible and that $\pi_{b}$ is more efficient than $\pi_{a}$. It is easily seen that the requirements of the QoS sensitive sessions are not violated under $\pi_{b}$ (that is $\pi_{b}$ is acceptable).

- For $t<{ }^{a} \mathrm{~b}_{k}$ : The NBSA bandwidth of the system is the same under the two policies [see (22)]. Sessions in ${ }^{a} \mathrm{I}_{k}^{P}$ are assigned the same weight under $\pi_{b}$ and $\pi_{a}$ so they get the same service under both policies, i.e., ${ }^{b} \hat{\mathrm{W}}_{i}(0, t)={ }^{a} \hat{\mathrm{W}}_{i}(0, t), \forall i \in{ }^{a} \mathrm{I}_{k}^{P}$. Sessions in ${ }^{a} \mathrm{I}_{k}^{F}$ are assigned a greater weight under $\pi_{b}$ than under $\pi_{a}$ so they get better service under $\pi_{b}$, i.e., ${ }^{b} \hat{\mathrm{W}}_{i}(0, t)>{ }^{a} \hat{\mathrm{W}}_{i}(0, t), \forall i \in{ }^{a} \mathrm{I}_{k}^{F}$. Sessions in ${ }^{a} \mathrm{I}_{k}$ are assigned a smaller weight under $\pi_{b}$ so they get worse service, i.e., ${ }^{b} \hat{\mathrm{W}}_{i}(0, t)<{ }^{a} \hat{\mathrm{W}}_{i}(0, t) \forall i \in{ }^{a} \mathrm{I}_{k}$, but by assumption they do not violate their delay bound.

- For ${ }^{a} \mathrm{~b}_{k}<t<{ }^{b} \mathrm{~b}_{k}$ : All sessions in ${ }^{a} \mathrm{I}_{k}^{P}$ have cleared their backlog under $\pi_{a}$ and $\pi_{b}$ so they get the same service under both policies, i.e., ${ }^{b} \hat{\mathrm{W}}_{i}(0, t)={ }^{a} \hat{\mathrm{W}}_{i}(0, t), \forall i \in$ ${ }^{a} \mathrm{I}_{k}^{P}$. Sessions in ${ }^{a} \mathrm{I}_{k}$ are assigned a smaller weight under $\pi_{b}$ than under $\pi_{a}$ so they get worse service, i.e., ${ }^{b} \hat{\mathrm{W}}_{i}(0, t)<{ }^{a} \hat{\mathrm{W}}_{i}(0, t) \forall i \in{ }^{a} \mathrm{I}_{k}$; but, by assumption they do not violate their delay bound. Sessions in ${ }^{a} \mathrm{I}_{k}^{F}$ are assigned a greater amount of work in $\left(0,{ }^{a} \mathrm{~b}_{k}\right]\left({ }^{b} \hat{\mathrm{W}}_{i}\left(0,{ }^{a} \mathrm{~b}_{k}\right)>{ }^{a} \hat{\mathrm{W}}_{i}\left(0,{ }^{a} \mathrm{~b}_{k}\right)\right)$, and at ${ }^{b} \mathrm{~b}_{k}$ the amount of work they have received is the same under both policies $\left({ }^{b} \hat{\mathrm{W}}_{i}\left(0,{ }^{b} \mathrm{~b}_{k}\right)={ }^{a} \hat{\mathrm{W}}_{i}\left(0,{ }^{b} \mathrm{~b}_{k}\right)\right)$. So, ${ }^{b} \hat{\mathrm{W}}_{i}(0, t)>{ }^{a} \hat{\mathrm{W}}_{i}(0, t)$ holds $\forall t \in\left({ }^{a} \mathrm{~b}_{k},{ }^{b} \mathrm{~b}_{k}\right), \forall i \in{ }^{a} \mathrm{I}_{k}^{F}$.

- For ${ }^{b} \mathrm{~b}_{k}<t$ : All sessions in ${ }^{a} \mathrm{I}_{k}^{P} \cup{ }^{a} \mathrm{I}_{k}$ have cleared their backlog under $\pi_{a}$ and $\pi_{b}$ so they get the same service under both policies, i.e., ${ }^{b} \hat{W}_{i}(0, t)={ }^{a} \hat{W}_{i}(0, t), \forall i \in$ ${ }^{a} \mathrm{I}_{k}^{P} \cup{ }^{a} \mathrm{I}_{k}$. The NBSA bandwidth of the system is the same under the two policies at ${ }^{b} \mathrm{~b}_{k}{ }^{+}$. This implies, in conjunction with (38), that sessions in ${ }^{a} \mathrm{I}_{k}^{F}$ are assigned the same amount of work under $\pi_{b}$ and $\pi_{a}$, i.e., ${ }^{b} \hat{\mathrm{W}}_{i}(0, t)=$ ${ }^{a} \hat{\mathrm{W}}_{i}(0, t), \forall i \in{ }^{a} \mathrm{I}_{k}^{F}$.

The best effort traffic is assigned a greater weight under $\pi_{b}$; the same conclusions as for the sessions in ${ }^{a} \mathrm{I}_{k}^{F}$ hold for it.

Finally, it is noted that since the exchange of $\phi$ s takes place in a continuous way, it is sufficient to show that the final policy is acceptable in order to ensure that every intermediate policy is acceptable. ${ }^{b} \mathrm{~b}_{k}$ can be replaced by any $\mathrm{t}^{a} \mathrm{~b}_{k}>t>{ }^{b} \mathrm{~b}_{k}$ and the proof that the intermediate policy is acceptable and more efficient still holds.

\section{B. Proof of Proposition 2 (Section III.A)}

According to Proposition 1, each time that the XMF process modifies the original policy the resulting policy $\left(\pi_{b}\right)$ is acceptable and more efficient. This implies that the generated by the XMF process policy is acceptable and more efficient than the original policy, except from the case where XMF does not modify the original policy.

Two policies are identical (all sessions are assigned the same weights under both policies) if and only if the backlog clearing time of each session in an all-greedy system is the same under both policies. In particular, in the all-greedy system ${ }^{a} \mathrm{~b}_{0}=0$ and ${ }^{a} \hat{\mathrm{C}}\left({ }^{a} \mathrm{~b}_{0}^{+}\right)=C_{G}$ hold under any policy $\pi_{a}$. If ${ }^{b} \mathrm{~b}_{1}={ }^{a} \mathrm{~b}_{1}=\mathrm{b}_{1}$ for two policies $\pi_{a}$ and $\pi_{b}$, it is concluded that ${ }^{b} \phi_{i}={ }^{a} \phi_{i}$ for all sessions $i$ for which $e_{i}=\mathrm{b}_{1}$ (where $e_{i}$ is defined as ${ }^{a} e_{i}=$ ${ }^{b} e_{i}=e_{i}$ for all sessions $i$ for which ${ }^{a} e_{i}={ }^{b} e_{i}$ ). If the same set of sessions empties its backlog at $b_{1}$, under both policies ${ }^{a} \hat{\mathrm{C}}\left(\mathrm{b}_{1}^{+}\right)={ }^{b} \hat{\mathrm{C}}\left(\mathrm{b}_{1}^{+}\right)$(the NBSA bandwidth has the same value right of the first backlog clearing time under both policies). If ${ }^{a} \mathrm{~b}_{2}={ }^{a} \mathrm{~b}_{2}=\mathrm{b}_{2}$, it is concluded that ${ }^{b} \phi_{i}={ }^{a} \phi_{i}$ for all sessions $i$ for which $e_{i}=\mathrm{b}_{2}$. If the same set of sessions empties its backlog at $\mathrm{b}_{2}$, under both policies ${ }^{a} \hat{\mathrm{C}}\left(\mathrm{b}_{2}^{+}\right)={ }^{b} \hat{\mathrm{C}}\left(\mathrm{b}_{2}^{+}\right)$holds. Similarly, for all backlog clearing times the validity of the claim becomes clear.

Now suppose that there were two policies, $\pi_{a 1}$ and $\pi_{a 2}$, for which $\operatorname{XMF}\left(\pi_{a 1}\right)=\pi_{o 1} \neq \pi_{o 2}=\operatorname{XMF}\left(\pi_{a 2}\right)$. Let ${ }^{o 1} \mathrm{I}_{m},{ }^{o 2} \mathrm{I}_{m}$ denote the set of sessions that empty their backlog $m$ th in order under $\pi_{o 1}$ and $\pi_{o 2}$, respectively. Suppose further that the two policies coincide for the set of sessions in ${ }^{o 1} \mathrm{I}_{n}={ }^{o 2} \mathrm{I}_{n}, n=0,1, \ldots, k-1$ and their first difference occurs for the sets ${ }^{o 1} \mathrm{I}_{k}{ }^{o 2} \mathrm{I}_{k}, k \geq 1$, i.e., the first backlog clearing time on which the two policies differ is considered. 
The differentiation between the two policies can have one of the following forms.

- ${ }^{o 1} \mathrm{I}_{k}={ }^{o 2} \mathrm{I}_{k}$, that is, under both policies the same sessions empty their backlog $k$ th in order, but they differ on the backlog clearing time of these sessions. Suppose ${ }^{o 1} \mathrm{~b}_{k}<{ }^{o 2} \mathrm{~b}_{k}$. This means that under $\pi_{o 1}$ sessions in ${ }^{o 1} \mathrm{I}_{k}$ are not compressible in $\phi$ space (else the XMF process would have expanded their busy periods) and under $\pi_{o 2}$ they are (contradiction).

- ${ }^{o 1} \mathrm{I}_{k} \neq{ }^{o 2} \mathrm{I}_{k}$. It is easily seen that a session which empties its backlog at a different time instance under the two policies exists. Such a session is not compressible in $\phi$ space under the one policy (the policy under which it empties its backlog first) and is compressible under the other (contradiction).

\section{REFERENCES}

[1] A. Parekh and R. Gallager, "A generalized processor sharing approach to flow control in integrated services networks: The single-node case," IEEE/ACM Trans. Networking, vol. 1, pp. 344-357, June 1993.

[2] A. Parekh and R. Gallager, "A generalized processor sharing approach to flow control in integrated services networks: The multiple node case," IEEE/ACM Trans. Networking, vol. 2, pp. 137-150, Apr. 1994.

[3] R. Szabó, P. Barta, F. Németh, and J. Biró, "Call admission control in generalized processor sharing (GPS) schedulers using nonrate proportional weighting of sessions," in Proc. IEEE INFOCOM, vol. 3, Mar. 2000, pp. 1243-1252.

[4] A. Elwalid and D. Mitra, "Design of generalized processor sharing schedulers which statistically multiplex heterogeneous QoS classes," in Proc. IEEE INFOCOM, Mar. 1999, pp. 1220-1230.

[5] Z. Zhang, D. Towsley, and J. Kurose, "Statistical analysis of the generalized processor sharing scheduling discipline," in Proc. ACM SIGCOMM, Sept. 1994.

[6] Z. Zhang, Z. Liu, J. Kurose, and D. Towsley, "Call admission control schemes under the generalized processor sharing scheduling," $J$. Telecommun. Syst., Modeling, Analysis, Design, Manage., vol. 7, no. 1, July 1997.

[7] Z. Zhang, Z. Liu, and D. Towsley, "Closed-form deterministic end-to-end performance bounds for the generalized processor sharing scheduling discipline," J. Combinatorial Optimization (Special Issue Scheduling), vol. 1, no. 4, 1998.

[8] J.-Y. Le Boudec, "Application of network calculus to guaranteed service networks," IEEE Trans. Inform. Theory, vol. 44, pp. 1087-1096, May 1998.

[9] L. Georgiadis, R. Guérin, V. Peris, and K. Sivarajan, "Efficient network QoS provisioning based on per node traffic shaping," IEEE/ACM Trans. Networking, vol. 4, pp. 482-501, Aug. 1996.

[10] G. Urvoy and G. Hébuterne, "An efficient weight assignment process for GPS servers," in Proc. ECUMN, 2000, pp. 171-178.

[11] O. Yaron and M. Sidi, "Generalized processor sharing networks with exponentially bounded burstiness arrivals," in Proc. INFOCOM, Jan. 1996, pp. 628-634.

[12] G. de Veciana and G. Kesidis, "Bandwidth allocation for multiple qualities of service using generalized processor sharing," IEEE Trans. Inform. Theory, vol. 42, pp. 268-72, Jan. 1996.

[13] K. Kumaran, G. E. Margrave, D. Mitra, and K. R. Stanley, "Novel techniques for the design and control of generalized processor sharing schedulers for multiple QoS classes," in Proc. IEEE INFOCOM, 2000.

[14] A. Kumar, M. Hegde, S. V. R. Anand, B. N. Bindu, D. Thirumurthy, and A. A. Kherani, Nonintrusive TCP connection admission control for bandwidth management of an internet access link, in IEEE Commun. Mag., May 2000.

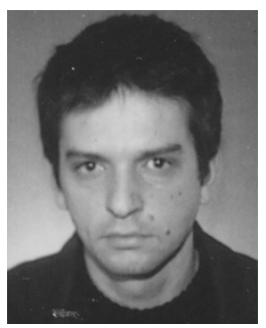

Antonis Panagakis received the B.Sc. degree in physics and the M.S. degree in electronics and telecommunications from the University of Athens, Athens, Greece, in 1996 and 1998, respectively. He is working toward the Ph.D. degree in telecommunications at the same university.

His research interests include provision of quality of service guarantees in wired and wireless networks.

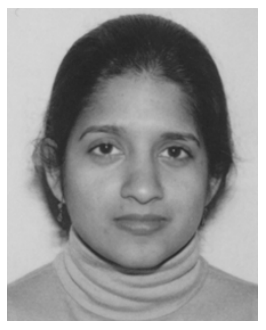

Nandita Dukkipati (S'01) received the B.Eng. degree in electronics and instrumentation from the Birla Institute of Technology and Science (BITS), Pilani, India, and the M.S. degree in electronics from the Indian Institute of Science, Bangalore, India, in 1998 and 2000, respectively. She is currently working toward the Ph.D. degree in electrical engineering at Stanford University, Stanford, CA.

Her research interests include congestion control in the Internet, provision of quality of service guarantees, and scheduling algorithms.

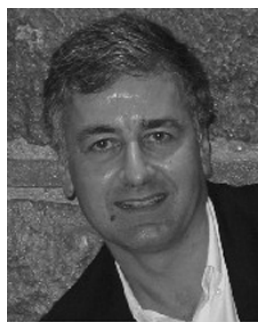

Ioannis Stavrakakis (S'84-M'88-SM'93) received the Diploma in electrical engineering from the Aristotelian University of Thessaloniki, Thessaloniki, Greece, in 1983 and the Ph.D. degree in electrical engineering from the University of Virginia, Charlottesville, in 1988.

He was an Assistant Professor of computer science and electrical engineering with the University of Vermont, Burlington, from 1988 to 1994, an Associate Professor of electrical and computer engineering with Northeastern University, Boston, MA, from 1994 to 1999, and is currently a Professor of informatics and telecommunications with the University of Athens, Athens, Greece. His teaching and research interests include resource allocation protocols and traffic management for communication networks, with recent emphasis on continuous media applications and ad hoc networking. His past research has been published in over 100 scientific journals and conference proceedings.

Dr. Stavrakakis is a a member of (and has served as an elected officer for) the IEEE Technical Committee on Computer Communications (TCCC) and the Chairman of IFIP WG6.3. He has served as a Co-organizer of the 1996 International Teletraffic Congress (ITC) mini-seminar, the Organizer of the 1999 IFIP WG6.3 workshop, a Technical Program Co-chair for the IFIP Networking2000 Conference, the Vice-General Chair for the Networking2002 Conference, and the Organizer of the COST-IST(EU)/NSF(USA)-sponsored NeXtworking'03. $\mathrm{He}$ is an Associate Editor for the IEEE/ACM TRANSACTIONS ON NETWORKING, the ACM/Baltzer Wireless Networks Journal, and the Computer Networks Journal. He has served repeatedly in NSF and IST research proposal review panels and is involved in the organization of numerous conferences sponsored by IEEE, ACM, ITC, and IFIP societies.

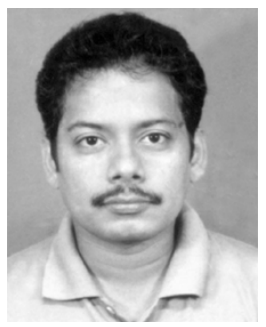

Joy Kuri received the Ph.D. degree from the Department of Electrical Communication Engineering, the Indian Institute of Science, Bangalore, India, in 1995.

He spent two years in Ecole Polytechnique, University of Montreal, and one and a half years in INRSTelecommunications, University of Quebec, Canada, as a Research Associate. Since 1999, he has been with the Centre for Electronics Design and Technology, Indian Institute of Science, where he is currently an Assistant Professor. His research and teaching interests include the areas of modeling, analysis, and control of communication networks and stochastic systems. 